\title{
Anti-Tumor and Anti-Invasive Effects of ONC201 on Ovarian Cancer Cells and a Transgenic Mouse Model of Serous Ovarian Cancer
}

\section{Yali Fan}

University of North Carolina at Chapel Hill Louis Round Wilson Special Collections Library: The University of North Carolina at Chapel Hill

\section{Jiandong Wang}

Capital Medical University

\section{Ziwei Fang}

University of North Carolina at Chapel Hill Louis Round Wilson Special Collections Library: The University of North Carolina at Chapel Hill

\section{Stuart R Pierce}

University of North Carolina at Chapel Hill Louis Round Wilson Special Collections Library: The University of North Carolina at Chapel Hill

\section{Lindsay West}

University of North Carolina at Chapel Hill Louis Round Wilson Special Collections Library: The University of North Carolina at Chapel Hill

\section{Allison Staley}

University of North Carolina at Chapel Hill Louis Round Wilson Special Collections Library: The University of North Carolina at Chapel Hill

\section{Katherine Tucker}

University of North Carolina at Chapel Hill Louis Round Wilson Special Collections Library: The University of North Carolina at Chapel Hill

\section{Yajie Yin}

University of North Carolina at Chapel Hill Louis Round Wilson Special Collections Library: The University of North Carolina at Chapel Hill

\section{Wenchuan Sun}

University of North Carolina at Chapel Hill Louis Round Wilson Special Collections Library: The University of North Carolina at Chapel Hill

\section{Weimin Kong}

Capital Medical University

\section{Varun Prabhu}

Oncoceutic.

\section{Joshua E Allen}


Oncoceutic

Chunxiao Zhou ( $\nabla$ czhou@med.unc.edu )

University of North Carolina at Chapel Hill

Victoria L Bae-Jump

University of North Carolina at Chapel Hill Louis Round Wilson Special Collections Library: The University of North Carolina at Chapel Hill

Research

Keywords: ONC201, DRD2, ovarian cancer, invasion, proliferation

Posted Date: January 25th, 2021

DOl: https://doi.org/10.21203/rs.3.rs-152449/v1

License: (c) (1) This work is licensed under a Creative Commons Attribution 4.0 International License. Read Full License

Version of Record: A version of this preprint was published at Frontiers in Oncology on March 17th, 2022. See the published version at https://doi.org/10.3389/fonc.2022.789450. 


\section{Abstract}

Background: ONC201 is a promising first-in-class small molecule that has been reported to have antineoplastic activity in various types of cancer through activation of tumor necrosis factor-related apoptosis-inducing ligand (TRAIL) as well as activation of mitochondrial caseinolytic protease P (ClpP).

Methods: Our objective was to evaluate the effect of the ONC201 on (1) proliferation, cellular stress, apoptosis and invasion in human serous ovarian cancer (OC) cell lines, and (2) inhibition of tumor growth in a genetically engineered mouse model of high grade serous OC (K18-gT121+/-;p53fl/fl;Brca1fl/fl; KpB) under obese (high fat diet) and lean (low fat diet) conditions.

Results: ONC201 significantly suppressed cell proliferation, induced arrest in G1 phase, and increased cellular stress and apoptosis, accompanied by dual inhibition of the AKT/mTOR/S6 and MAPK pathways in OC cells. ONC201 also resulted in inhibition of adhesion and invasion via epithelial-mesenchymal transition and reduction of VEGF expression. Pre-treatment with the anti-oxidant, N-acetylcysteine (NAC), reversed the ONC201-induced oxidative stress response, and prevented ONC201-reduced VEGF and cell invasion by regulating epithelial-mesenchymal transition protein expression. Knockdown of ClpP in ovarian cancer cells reduced ONC201 mediated the anti-tumor activity and cellular stress. Diet-induced obesity accelerated ovarian tumor growth in the KpB mouse model. ONC201 significantly suppressed tumor growth, and decreased serum VEGF production in obese and lean mice, leading to a decrease in tumoral expression of Ki-67, VEGF and phosphorylation of p42/44 and S6 and an increase in ClpP and DRD5, as assessed by immunohistochemistry. Additionally, ONC201 exhibited greater anti-tumor efficacy in obese $(75 \%)$ as compared to lean (65\%) mice. Interpretation

Conclusions: These results suggest that ONC206 may be a promising therapeutic agent to be explored in future clinical trials in high grade serous OC.

\section{Background}

Ovarian cancer $(\mathrm{OC})$ is the second most common gynecological cancer and the most lethal gynecologic malignancy in the United States, with an estimated 21,750 new cancer cases and 13,940 cancer deaths in 2020 (1). At least $70 \%$ of patients are diagnosed at advanced stages, and no specific symptoms or diagnostic tools are currently available for early diagnosis(2). Despite treatment with aggressive debulking surgery and combination chemotherapy (carboplatin and paclitaxel), up to $75 \%$ of patients with advanced OC experience tumor progression or recurrence, with a dismal 5-year overall survival (OS) of $25 \%$, largely due to the emergence of drug resistance(2-4). Therefore, the identification of novel target molecules and the development of new therapeutic agents are desperately needed to improve outcomes for this highly lethal disease. 
ONC201 is a promising first-in-class small molecule that was identified in a high-throughput phenotypic cell-based screen as an efficacious anti-tumorigenic therapeutic agent(5). The mechanism of action of ONC201 results from stimulation of activating transcription factor 4 (ATF4) as well as the C/EBP homology protein (CHOP)-mediated integrated stress response (ISR), ultimately leading to induction of death receptor 5 (DR5) and tumor necrosis factor-related apoptosis-inducing ligand (TRAIL) and inhibition of Akt/ERK signaling pathways $(5,6)$. More recent studies found that mitochondrial caseinolytic protease $\mathrm{P}(\mathrm{ClpP})$ is another critical target for ONC201, and activation of ClpP by ONC201 results in modulation of the ISR pathway, inhibition of protein synthesis and induction of mitochondrial dysfunction, which ultimately leads to inhibition of tumor growth in vitro and in vivo(7, 8). Pre-clinical studies have shown that ONC201 has anti-proliferative and anti-tumorigenic effects in solid tumors and hematologic malignancies $(6,9,10)$. We recently found that ONC201 exhibited anti-tumorigenic and antimetastatic activity in uterine serous carcinoma (USC) in vitro (11). Phase I clinical trials showed that ONC201 is clinically active and exceptionally well-tolerated with favorable pharmacokinetics and pharmacodynamics in a variety of cancers, including lymphoma, glioblastoma and endometrial cancer(5, 11-15). Currently, multiple phase II clinical trials are underway to evaluate the single agent therapeutic efficacy of ONC201, including trials in endometrial and OC $(5,16)$.

Given that ONC201 has unique features including broad-spectrum activity independent of mutations or tumor type, oral administration and excellent safety profile, our objective was to evaluate the effect of ONC201 on cell proliferation, cellular stress, apoptosis, invasion and tumor growth in OC cell lines and a genetically engineered mouse model of high grade serous OC; given the inter-relationship between mitochondrial dysfunction and obesity as well as ONC201's impact on the mitochondrial modulator ClpP, we studied the anti-tumorigenic effects of ONC201 in both obese and lean mice with OC. Our results show that ONC201 demonstrates promise as a single targeted agent for OC, worthy of further evaluation in clinical trials.

\section{Methods}

\section{Cell culture and reagents}

Four ovarian cancer cell lines, OVCAR3, OVCAR5, IGROV-1 and SKOV3, were used in this study. The cells were grown in DMEM/F12(1:1) supplemented with 10\% fetal bovine serum, 2 mM L-glutamine, 10,000 $\mathrm{U} / \mathrm{ml}$ penicillin and $10,000 \mu \mathrm{g} / \mathrm{ml}$ streptomycin under $5 \% \mathrm{CO}_{2}$. ONC201 was kindly provided by Oncoceutics, Inc. MTT, propidium iodide and RIPA buffer were purchased from Sigma (St. Louis, MO). Antibodies to PERK, ATF4, CHOP, BCL-XL, MCL-1, PARP, DR5, $\beta$-actin, VEGF, Slug, Snail, CyclinD1, CDK4, and CDK6 were obtained from Cell Signaling Technology (Beverly, MA). DRD2(B-10):sc-5303 and DRD5(E12):sc-376088 were purchased from Santa Cruz Biotechnology. The TMRE and JC-1 probes were purchased from AAT bioquest (Sunnyvale, CA).

\section{Cell proliferation assay}


The four OC cell lines were incubated in 96-well plates (4000 cells/well) in the presence of varying concentrations of ONC201 for 72 hours. 5 ul MTT $(5 \mathrm{mg} / \mathrm{mL})$ was added in each well following by incubation for $1-2$ hours at $37^{\circ} \mathrm{C}$. MTT absorbance was measured at $575 \mathrm{~nm}$ after $100 \mathrm{ul}$ DMSO was added to the plates. Effects of ONC201 on cell proliferation were assessed as a percentage of control cell proliferation obtained from 1\% PBS treated cells grown in the same 96-well plates followed by IC50 analysis.

\section{Colony formation assay}

The OVCAR5 and SKOV3 cells were plated at a density of 200 cells in 6-well plates in triplicated for each treatment group for 24 hours. The cells were treated with indicated concentrations of ONC201 for 30 hours and then incubated at $37^{\circ} \mathrm{C}$ for 10-14 days. The culture medium was changed every third or fourth day. The cells were fixed in methanol and stained with $0.5 \%$ crystal violet. The colonies that contain more than 50 cells were counted under the microscope.

\section{Cell cycle analysis}

The cells were grown in in 6-well plates overnight and then incubated with varying concentrations of ONC201 for 36 hours. Cells were harvested, washed with PBS, fixed in $2 \mathrm{ml}$ of ice-cold $90 \%$ ethanol and stored overnight at $-20^{\circ} \mathrm{C}$ until cell cycle analysis was performed. On the day of analysis, the cells were resuspended in $100 \mathrm{ul}$ RNase $A$ solution containing propidium iodide $(2 \mathrm{mg} / \mathrm{ml})$ for $30 \mathrm{~min}$ at room temperature in the dark. DNA content was determined by Cellometer (Nexcelom, Lawrence, MA). Cell cycle was analyzed using FCS4 express software (Molecular Devices, Sunnyvale, CA).

\section{Annexin V assay}

Cell apoptosis was determined using Annexin-V FITC kit (BioVision, Mountain View, CA) following manufacture' protocol. The cells were seeded into 6 well plates at a density of $2.5 \times 10^{5} \mathrm{cells} /$ well and then treated with varying concentrations of ONC201 for 30 hours. The cells were harvested and stained with 100 ul of Annexin-V and PI dual-stain solution for 15 min in the dark. Annexin $V$ expression was determined by a Cellometer. Apoptosis cells were analyzed by FCS4 express software.

\section{Caspase 3, 8 and 9 Assays}

Caspase activity assays were performed with modifications as previously described(17). In brief, the OVCAR5 and SKOV3 cells were seeded in 6-well plates at a density of $2.5 \times 10^{5}$ cells/well overnight. The cells were treated with ONC201 at different concentrations for 24 hours. 150-180 ul 1X caspase lysis buffer was added to each well. BCA protein assay was used for quantitation of protein concentration. 1015 ug lysates in a black clear bottom 96-well plate were incubated with reaction buffer and $200 \mathrm{uM}$ of 
caspase substrates for $30 \mathrm{~min}$. The fluorescence of each well was determined using a microplate reader (Tecan, Morrisville, NC). The selective substrates Ac-DEVD-AMC, Ac-IETD-AFC and Z-IETD-AFC (AAT Bioquest) were used for caspase 3, caspase 8 and caspase 9, respectively. Each experiment was repeated three times to assess for consistency of results.

\section{Reactive oxygen species (ROS) assay}

ROS production was determined using the DCFH-DA assay, as previously described (18). The OVCAR5 and SKOV3 cells (8000 cells/well) were cultured in a black 96 -well plate overnight followed by treatment with indicated doses of ONC201 for 12 hours. Cells were then incubated with 20 uM DCFH-DA in regular growth medium for 30 minutes. ROS accumulation was measured by a plate reader (Tecan) at an excitation wavelength of $485 \mathrm{~nm}$ and an emission wavelength of $530 \mathrm{~nm}$.

\section{Mitochondrial membrane potential assay}

Mitochondrial membrane potential was analyzed using the specific fluorescent probes JC- 1 and TMRE, respectively $(19,20)$. The cells were cultured overnight in a 96 well plate and then treated with different concentrations of ONC201 for 8 hours. Treated cells were incubated with 2 uM JC-1 or 1 uM TMRE for 30 minutes at $37^{\circ} \mathrm{C}$. The levels of the fluorescent probes were measured using a Tecan plate reader at $\mathrm{Ex} / \mathrm{Em}=549 / 575 \mathrm{~nm}$ for TMRE. For JC-1, green JC-1 signals were measured at Ex/Em 485/535 nm and red signals were measured at 535/590 nm. Each experiment was repeated three times to assess for consistency of results.

\section{Adhesion assay}

Cell adhesion was measured by using laminin adhesion assay as previously described (11). Briefly, a 96well plate was coated with $100 \mathrm{ul} \mathrm{laminin}-1(10 \mathrm{ug} / \mathrm{ml})$ for 1 hour and was blocked by $3 \%$ BSA for 30 min at $37^{\circ} \mathrm{C}$. The cells were pre-treated with ONC201 for 24 hours and then added to the laminin coated wells (25,000 cells). The plate was incubated at $37^{\circ} \mathrm{C}$ for 1 hour in serum-free medium. The cells were washed twice with PBS to remove nonadherent cells. Attached cells were fixed by adding 100 ul of $5 \%$ glutaraldehyde for 20-30 min and stained with $0.1 \%$ crystal violet for $30 \mathrm{~min}$. Next, the cells were lysed with $10 \%$ acetic acid and the absorbance of the solution was measured at $570 \mathrm{~nm}$ in a plate reader (Tecan).

\section{Transwell assay}

Cell invasion assays were performed using a 96 well plate coated with 0.5-1 x BME. The OVCAR5 and SKOV 3 cells were starved for 12 hours and then seeded in the upper chambers of the wells and the lower chambers were filled with regular medium and differing concentrations of ONC201. The plates were incubated for 3 hours to allow invasion into the lower chambers. After washing the upper and lower 
chambers with PBS, 100 ul of calcein AM solution (Invitrogen, Carlsbad, CA) was added to the lower chambers and incubated for 30-60 minutes. The lower chamber plate was measured by plate reader for reading fluorescence at EX/EM 485/520 nm. Each experiment was repeated twice

\section{Wound healing assay}

The cells were plated in 6 well plates at $3.5 \times 10^{5}$ cells/well for 24 hours and then replaced with media with $0.5 \%$ charcoal stripped FBS for 12 hours. A sterilized 200 ul pipette tip was used to draw a straight line across the plate in one direction. The cells were then washed with fresh media to remove the detached cells, and cells were then treated with different concentrations of ONC201 in the media supplemented with $0.5 \%$ charcoal stripped serum for 24 to 48 hours. The images were acquired at different timing points $(24,36$ and 48 hours). Measurements of the width of the wound were performed at random intervals with the Adobe Photoshop CS6.

\section{Organotypic 3D co-cultures}

The organotypic culture was performed as previously described(21). $1 \times 10^{6}$ mouse stromal cells transfected with hTERT were mixed with 3.5 volumes of Matrigel $\circledast$, 1 volume of 10X DMEM, 1 volume of FCS, and 1 volume of DMEM/F12, and then were pipetted into each well of a 24-well plate. After 18 hours, $5 \times 10^{5}$ OVCAR5 cells, suspended in $1 \mathrm{ml}$ regular media supplemented with $10 \% \mathrm{FCS}$, were added to each well. The OVCAR5 cells were treated with 10 uM ONC201 for 36 hours. Then the gel with stromal and cancer cells was lifted and placed onto a stainless steel grid in a 6 well plate. DMEM/F12 media was added to the well so that the gel plug was exposed to the air from above and to the media from below. The media was changed every 3 days maintaining the air-liquid interface. The organotypic gels were cultured for 12 to 14 days and then fixed in $4 \%$ formaldehyde for 24 hours. The gels were bisected and processed to paraffin blocks. Each slide was stained using standard haematoxylin and eosin (H\&E), and then scanned by Motic. The "Invasion Index" was calculated by ImagePro as MCY $\times N \times A$ (MCY: Mean Cord $\mathrm{Y}, \mathrm{N}$ : the number of cancer islands, $\mathrm{A}$ : sum of areas of the cancer islands). The gels not treated with ONC201 were used as control.

\section{Transient ClpP knockdown}

The OVCAR5 and SKOV3 cells were transiently transfected with 40 pmol of either siRNA targeting ClpP and $40 \mathrm{pmol}$ of scrambled control siRNA (Sigma) using Mission SiRNA Transfection Reagent, according to manufacturer's protocol. MTT assay were performed at 48 hours post-transfection. Western blotting were performed at 24 houea after transfection.

\section{Western immunoblotting}


The OVCAR5 and SKOV3 cells were treated with ONC201 for 30 hours. Total cell lysates were prepared in RIPA buffer plus PhosStop. Protein concentration was quantified using BCA assay (Sigma). Equal amounts of lysates were electrophoresed on 10-12 \% SDS-PAGF and transferred onto a PVDF membrane. The membranes were probed at $4^{\circ} \mathrm{C}$ overnight with appropriate primary antibodies. Proteins were visualized using SuperSignal West Pico Substrate (Thermo Scientific) by the ChemiDoc image system (Bio-Rad).

\section{ONC201 treatment in KpB mouse model}

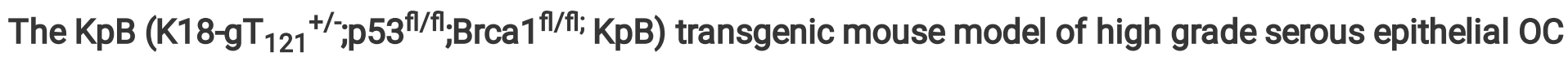
has been described previously in detail (22-24). Animal protocol was approved by the UNC-CH Institutional Animal Care and Use Committee (IACUC). Mice were housed on a 12 hours light, 12 hours dark cycle, with free access to food and water The KpB female mice were fed a high fat diet (HFD, 60\% calories from fat) or a low fat diet (LFD, 10\% calories from fat, Research Diets) at 3 weeks of age. 5 ul of $2.5 \times 10^{7}$ P.F.U of recombinant adenovirus Ad5-CMV-Cre (AdCre, Transfer Vector Core, University of lowa) was injected into the left ovarian bursa cavity at 6-8 weeks age(23). The mice were checked weekly by abdominal palpation for the appearance of ovarian tumors. Once tumors had reached an average size of $0.1 \times 0.1 \mathrm{~cm}$ in diameter by palpation, the mice fed with HFD or LFD ( $N=15 /$ group) were assigned into the different groups: ONC210 was given weekly in $0.1 \mathrm{ml}$ containing either $130 \mathrm{mg} / \mathrm{kg}$ or placebo in oral gavage for 4 weeks. The size of ovarian tumors were measured twice a week using palpation. The mice were weighted weekly and observed daily any signs of toxicity or distress. After 4 weeks of treatment, mice were sacrificed by $\mathrm{CO} 2$ asphyxiation and tumors were weighted. Ovarian tumor volumes were calculated as following: (width $2 \times$ length)/ 2 .

\section{Immunohistochemical analysis}

Five micrometer paraffin sections from the KpB mice tumors were processed for IHC analysis at IHC Mice Core Facility at UNC. Briefly, the slides were incubated with Ki-67, phosphorylated-42-44, phosphorylatedS6, and VEGF at $4^{\circ} \mathrm{C}$ overnight, respectively, and then treated with HRP-conjugated antibody for 1 hour. The sections were further applied with ABC-Staining Kits (Vector Labs, Burlingame, CA) for color reaction and hematoxylin for counterstaining. All IHC slides were scanned by Motic and scored by ImagePro software (Rockville, MD).

\section{Serum VEGF assay}

The VEGF productions of mice serum after treatment with ONC201 were detected using a VEGF ELISA Kit (R\&D Systems, Minneapolis, MN), according to the manufacturer's directions. Each sample from 0NC201 and control groups was measured in duplicate. Plates were read at $570 \mathrm{~nm}$ using a Tecan plate reader. 


\section{Statistical analysis}

Data are given as the mean \pm SD. Statistical significance was analyzed by the two-sided unpaired Student's t-test from at least three replicates. Tumor growth in different treatment arms was analyzed by One-way \& Two-way ANOVA test. GraphPad Prism 5 (La Jolla, CA USA) was used for all graphs and significance tests. $P$ values of $<0.05$ were considered to have significant group differences.

\section{Results}

\section{ONC201 inhibited cell viability in OC cell lines}

ONC201-mediated inhibition of OC cell viability was assessed using MTT assay. The OVCAR3, IGROV-1, OVCAR5 and SKOV3 were cultured in media with various concentrations of ONC201 for 72 hours. The MTT results showed that with increasing of ONC201, a dose-dependent growth inhibition was observed in four OC cell lines compared to the control cells (Figure 1A). The mean IC50 values of ONC201 were 4.2 UM for OVCAR3, 3.1 uM for IGROV-1, 3.2 uM for OVCAR5 and 2.1 uM for SKOV3. Subsequently, because colony formation assay is a well-established in vitro technique for testing the proliferative capability of treated cells (25), we investigated the long-term effect of ONC201 on OC cell growth. The OVCAR5 and SKOV3 cells were seeded in same density and incubated 10 uM of ONC201 for 2 days and subsequent culture of the cells for 12 days. The results showed that the colony-forming ability was reduced by $58.3 \%$ in OVCAR5 and $57.5 \%$ in SKOV3, respectively (Figure 1B). These results suggest that OC cells are sensitive to the anti-proliferative effects of ONC201.

Because ONC201 is a selective antagonist of the G protein-coupled receptor DRD2 that causes p53independent apoptosis through upregulation of TRAIL and DR5 in tumor cells (5), we next detected the effect of ONC201 on DRD2, DRD5 and DR5 in the OVCAR5 and SKOV3 cells. The cells were treated with ONC201 at 1, 10 and 100 uM for 24 hours. Western blotting results showed that ONC201 treatment significantly increased the expression of the DR5 protein and decreased the expression of DRD2 in a dose-dependent manner in both cell lines (Figure 1C).

\section{ONC201 caused cell cycle G1 arrest in OC cells}

We next investigated whether ONC201 modulates cell cycle progression in OC cells. As illustrated in Figure $2 A$ and B, treatment with 1, 10 and 100 uM ONC201 for 36 hours caused significant increases in the $\mathbf{G 1}$ phase and decreases $\mathrm{S}$ phase in a dose-dependent manner in the OVCAR5 and SKOV3 cells. G1 arrest phase increased from $44.69 \%$ in control cells to $60.12 \%$ in the 100uM ONC201-treated OVCAR5 cells and 56.89 to $71.55 \%$ in the SKOV3 cells; in parallel, the S phase cell population decreased from 25.41 to $15.62 \%$ with increasing concentrations of ONC201 in the OVCAR5 and 21.2 to $13.93 \%$ in the SKOV 3 cells $(p<0.05)$. To further understanding the mechanism underlying the cell cycle arrest, cell cyclerelated proteins were analyzed by western blotting in the ONC201-treated OVCAR5 and SKOV3 cells. The 
results showed that ONC201 resulted in reduced level of cyclin D1 expression in both cell lines after 36 hours of treatment. Moreover, ONC201 also inhibited the expression of the cyclin D1 regulatory partners, CDK4 and CDK6, following ONC201 treatment for 30 hours (Figure 2C). These data suggest that ONC201 induces cell cycle $\mathrm{G} 1$ arrest through cyclin D1 degradation in $\mathrm{OC}$ cells.

\section{ONC201 induced apoptosis in OC cells}

To characterize the underlying mechanism of growth inhibition by ONC201, the apoptotic cells were analyzed by performing an Annexin-V assay after treating the OVCAR5 and SKOV3 cell lines with ONC201 (1-100 uM). Annexin- $V$ analysis showed significant increases in apoptotic cells in a dose-dependent manner after 30 hours of ONC201 treatment, along with decreasing expression of MCL-1 and BCL-XL protein (Figure $3 A$ ). Annexin V expression increased from $6.61 \%$ in control cells to $19.15 \%$ in the $100 \mathrm{uM}$ ONC201-treated OVCAR5 cells and 6.68 to $16.12 \%$ in the SKOV3 cells. To examine whether ONC201 induces apoptosis through the mitochondrial apoptosis pathways in the $\mathrm{OC}$ cells, western blotting results showed that ONC201 significantly induced cleaved PARP and caspase 9 protein expressions in both cell lines (Figure 3B). Furthermore, a dose-dependent increase in the activity of cleaved caspase 3, 8 and 9 was found by ELISA assays in OVCAR5 and SKOV3 cells in response to ONC201 treatment (Figure 3C). These results indicated that apoptosis induced by ONC201 was executed through activation of either the extrinsic pathway (death receptor) or the intrinsic pathway in OC cells.

\section{ONC201 inhibited tumor growth in a transgenic mouse model of OC}

Given that obesity is associated with worse outcomes for ovarian cancer and dopamine signaling pathway contributes to the distinct metabolic profiles of obese and non-obese patients, we sought to evaluate whether ONC201 was able to inhibit tumor growth in a transgenic mouse model of high grade serous $\mathrm{OC}(\mathrm{KpB})$ under obese and non-obese conditions. Immune competent mice carrying Cre-inducible oncogenic Brca1 in combination with deletion of p53 and Rb in the ovaries develop high grade serous OC about 4-6 months after injection with AdCre (24). The HFD-fed or LFD-fed KpB mice were divided into four groups: HFD, HFD+ONC201, LFD and LFD+ONC201. The mice were treated once a week by oral gavage with either ONC201 $(130 \mathrm{mg} / \mathrm{kg}, 4$ weeks) or placebo after tumor induction. The tumor size was monitored twice a week by palpation. The mice showed tolerance to ONC201 treatment and did not show any obvious changes in behavior and body weight. After 4 weeks of treatment, the tumors were excised, weighed, and examined histologically. ONC201 effectively inhibited tumor growth and reduced tumor weight in both HFD and LFD groups compared to control groups. Ovarian tumor weights in obese KpB mice were significantly greater than that in the non-obese control mice ( $2.92 \mathrm{~g}$ versus $1.80 \mathrm{~g}$ ), suggesting that obesity promoted ovarian tumor growth. ONC201 treatment decreased tumor weight by $75.5 \%$ in obese mice and $65.2 \%$ in non-obese mice compared to their control groups (Figure 4A-C). ONC201 caused a greater anti-tumor effect in obese mice compared with control mice and, in non-obese mice. These 
findings imply that ONC201 caused a greater anti-tumor effect in obese mice compared to non-obese KpB mice although gene expression and metabolomic profiling showed statistically significant differences between the ovarian tumors from the obese versus lean mice(26). Collectively, these data demonstrate that treatment with ONC201 significantly suppressed OC growth in a genetically engineered mouse model of $\mathrm{OC}$ under obese and non-obese conditions.

To determine the anti-tumorigenic mechanisms of ONC201 in vivo, the expression of Ki-67 and DRD5 in ovarian tumors was evaluated by $\mathrm{IHC}$ in obese and non-obese $\mathrm{KpB}$ mice after 4 weeks of treatment. The tumors from obese mice displayed increased expression of the Ki67 and DRD5 compared to non-obese mice. Consistent with our results in vitro, ONC201 inhibited Ki-67 expression in the ONC201-treated obese mice by $33.6 \%$ and the ONC201-treated lean mice by $26.1 \%$. We also found that the expression of DRD5 was reduced in the obese and lean mice treated with ONC201 but not in the placebo-treated mice (Figure 4D), suggesting that ONC201 inhibits ovarian tumor growth through the DRD5 pathway.

\section{ONC201 induced cellular stress in OC cells}

ROS have been implicated as mediators of TRAIL-induced apoptosis in cancer cells via different pathways (27). To examine the involvement of oxidative stress in the anti-tumorigenic effect of ONC201 in OC cells, intracellular ROS levels were detected using the DCFH-DA assay. The results showed a dosedependent increase in ROS production when OVCAR5 and SKOV3 cells were treated with ONC201 at different concentrations for 12 hours. At a concentration of $100 \mathrm{uM}, 0 \mathrm{NC2} 21$ significantly increased DCFH-DA fluorescence 1.49 and 1.30-fold in OVCAR5 and SKOV3 cells ( $p<0.01$ ), respectively (Figure 5A).

To further evaluate the underlying mechanism of ROS effect in association with mitochondrial function, we next set out to assess the ability of ONC201 to depolarize mitochondrial membranes by JC-1 and TMRE ELISA assays. JC-1 assay showed that ONC201 induced the loss of mitochondrial transmembrane potential $(\Delta \Psi \mathrm{m})$ in both cell lines after 8 hours of treatment compared to control cells. ONC201 at $10 \mathrm{uM}$ significantly reduced mitochondrial transmembrane potential by $20.8 \%$ and $23.5 \%$ in the OVCAR5 and SKOV3 cells $(p<0.01)$, respectively (Figure $5 B$ ). Similarly, these changes of $\Delta \Psi \mathrm{m}$ in response to treatment were also observed in a TMRE assay in both cell lines (Figure 5C), which further strengthens the reliability of our results. Moreover, western blotting analysis showed that ONC201 significantly increased expression of mitochondrial protease, ClpP, and endoplasmic reticulum (ER) stress-related markers including ATF4, PERK, CHOP and IRE-1a in a dose-dependent manner in both cell lines after 24 hours of treatment (Figure 5D).

ClpP is essential for the oxidative stress response, and ONC201 is an allosteric agonist of ClpP. To understand the effect of ClpP on ONC201 mediated cell proliferation and cell stress, OVCAR5 and SKOV3 cells were transfected with ClpP siRNA or scramble RNA, respectively. As shown in Figure 5E, the protein expression of ClpP was downregulated significantly by ClpP siRNA in both cells when compared to the cells transfected with mock siRNA, which was accompanied by a decrease in the expression of PERK and phosphorylation S6, indicating mTOR/S6 signaling pathway might be involved in ClpP induced stress 
response. The siRNA-mediated knockdown of ClpP effectively inhibited the expression of ATF4 and PERK induced by ONC201 (Figure 5F). ONC201 mediated inhibition of cell proliferation in OVCAR5 and SKOV3 cells partially recovered by transfection with ClpP siRNA (Figure 5G). Importantly, IHC results confirmed ONC201 significantly increased the expression of ClpP in obese and lean mice after 4 weeks of treatment (Figure 5H). These results suggest that the anti-tumorigenic effects of ONC201 are in part depend on ClpP activated the integrated stress response in OC.

\section{ONC201 inhibited adhesion and invasion in OC cells and ovarian tumors}

Given that ONC201 exhibited anti-invasive ability in uterine serous carcinoma cells (11), we investigated the impact of ONC201 on cell adhesion, migration and invasiveness in the OVCAR5 and SKOV3 cell lines. In the assessment of cell adhesion, both cell lines were incubated in laminin-coated 96 well plates and treated with ONC201 for 2 hours. As shown in Figure 6A, cellular adhesion was decreased by $61.6 \%$ to $35.1 \%$ in the SKOV3 and OVCAR5 cells, respectively, at a dose of $100 \mathrm{uM}$. Cell invasion was measured using a transwell invasion assay with a Matrigel-coated filter. Both cell lines were seeded in the upper chambers of the transwell and treated with ONC201 (1-100 uM) for 4 hours. The invasive capacity of the OVCAR5 and SKOV3 cell lines was reduced by ONC201 treatment in a dosedependent manner. ONC201 (100 uM) significantly reduced the invasive ability of the OVCAR5 and SKOV3 cell lines by $23.3 \%$ and $36.0 \%$ (Figure 6B).

To evaluate the effect of ONC201 on cell migration, a wound-healing assay was performed in OVCAR5 and SKOV3 cells. The cells were treated with different concentrations of ONC 201 and evaluated the cell migration capacity at 24, 48 and 72 hours. ONC201 had a significant inhibitory effect on cell migration of OVCAR5 and SKOV3 cells at all times compared with vehicle-treated control cells $(p<0.05)$, which was most pronounced at 48 hours of treatment as shown in Figure $6 \mathrm{C}$.

Given that stromal cells have an active role in inducing EMT and enhancing invasive potential, and the pattern of invasion produced in organotypic cultures displays a similar invasion patterns observed in human(28), we used organotypic cultures containing stromal cells to measure the invasion capacity of OC cells after treatment with ONC201. The organotypic gels stained with H\&E were analyzed to generate an invasion index(29). Similar to wound healing and transwell assays, 14 days after treatment, addition of 10 uM ONC201 for 24 hours significantly reduced the invasion index, and OVCAR5 cells invasion was inhibited by $39.3 \%$ in organotypic cultures compared to control (6D).

We next examined the effect of ONC201 on the EMT and angiogenesis in the OC cells. Treatment with ONC201 for 24 hours significantly decreased the expression of VEGF-C, Slug and Snail in both cell lines (Figure 6E). IHC results showed that obesity increased VEGF expression in the ovarian tumors, when comparing tumors from obese versus lean mice. However, ONC201 reduced VEGF expression by $42.5 \%$ in the obese mice and by $42.2 \%$ in the lean mice as compared to controls (Figure 6F, p<0.05). In addition, we 
found that the production of serum VEGF decreased by $14.0 \%$ in obese mice and $19.4 \%$ in lean mice in comparison to placebo-treated mice (Figure 6G). Together, these findings support the contention that ONC201 has an ability to inhibit adhesion, invasion and angiogenesis in OC cells and ovarian tumors in $\mathrm{KpB}$ mice.

\section{Inhibition of cellular stress reduced the effects of ONC201 on cell proliferation and invasion.}

Girven that ONC201 is a potent activator of the ClpP-induced integrated stress response, we investigated the role of cellular stress in ONC201's anti-proliferative and anti-invasive effects. The OVCAR5 and SKOV3 cells were treated with ONC201 for 72 hours in the presence and absence of $1 \mathrm{mM}$ of the antioxidant, $\mathrm{N}$ acetylcysteine (NAC). The results showed that NAC partially reversed the cytotoxic effects of ONC201 in both cell lines (Figure 7A and B). Similarly, pre-treatment of NAC for 6 hours effectively reversed ONC201induced decreases in mitochondrial membrane potential and inhibited ONC201-induced increases in intracellular ROS levels (Figure 7B and C). These results suggest that cellular stress is essential for the anti-proliferative activity of ONC201 in OC cells.

Because NAC significantly inhibited ONC201-induced oxidative stress, efforts were made to explore whether ONC201 exerted anti-invasive effects through oxidative stress pathways. The wound healing assay was used to detect the ability of invasion after ONC201 treatment in both cell lines. In the presence of $10 \mu \mathrm{M}$ or $100 \mu \mathrm{M}$ ONC201, pre-treatment with NAC for 6 hours partially prevented ONC201-induced anti-invasive activity by $43.5 \%$ and $35.0 \%$ in the OVCAR5 cells, and $29.2 \%$ and $36.2 \%$ in the SKOV 3 cells, respectively (Figure 7D, $p<0.01$ ). Western blotting results indicated that $1 \mathrm{mM} \mathrm{NAC}$ treatment did not change the expression of CLPP induced by ONC201. However, in NAC-treated groups, NAC partially blocked $10 \mu \mathrm{M}$ ONC201-evoked decreases in VEGF and Snail expression (Figure 7E). Therefore, it appears that ONC201 exerted its anti-proliferative and anti-invasive effects partially through the integrated stress response in $\mathrm{OC}$ cells.

\section{ONC201 inhibited P13K/AKT/mTOR and MAPK pathways in vitro and in vivo}

To gain insight into the role of ONC201 in TRAIL-mediated signaling, we evaluated whether $\mathrm{P} 13 \mathrm{~K} / \mathrm{AKT} / \mathrm{mTOR}$ and MAPK pathways were involved in the anti-proliferative effects of ONC201 in OC cells. OVCAR5 and SKOV3 cells were treated with ONC201 (1, 10 and $100 \mathrm{uM})$ for 24 hours. mTOR activity was determined by phosphorylation of S6 (Ser235/236) and MAPK activation by detecting P42/44 phosphorylated on Thr202 and Tyr204. ONC201 inhibited AKT phosphorylation and activity of mTOR and MAPK pathways without changing the total levels of S6, p42/44 and AKT proteins in the both cells (Figure 8A). The effects of ONC201 on the phosphorylation-dependent activation of p42/44 and S6 in the KpB serous OC mouse model are shown in Figure 8B. ONC201 reduced p42/44 phosphorylation by 
$24.3 \%$ in obese mice and $38.8 \%$ in lean mice, respectively $(p<0.05)$. Similarly, ONC201 also reduced phosphorylation of $\mathrm{S} 6$ by $46.1 \%$ and $37.3 \%$ in obese and lean mice, respectively, compared to untreated mice. Overall, these data confirm that ONC201 reduces cell growth via inhibition of the AKT/mTOR and MAPK signaling pathways in $\mathrm{OC}$ cells and tumors.

\section{Discussion}

The heterogeneous expression of dopamine receptors in different types of cancer cells suggests that these receptors may exhibit varying functions to either stimulate or inhibit cancer cell growth $(30,31)$. TCGA data showed that DRD2 is elevated in several types of human cancer including OC, and its expression tends to correlate with progression and prognosis in some cancers, such as gastric cancer and neuroendocrine tumors $(32,33)$. Targeting DRD2 by RNAi or antagonists significantly reduces the viability of cancer cells and slows tumor growth in vivo in pancreatic and OC pre-clinical models $(34,35)$. ONC201 is the first selective antagonist of D2-like dopamine receptors for clinical oncology $(10,36)$. Our recent study showed that ONC201 targeted DRD2 and reduced cell growth and migration in USC cells (11). In this study, we investigated the impact of the anti-tumorigenic activity of ONC201 in human OC cell lines and the $\mathrm{KpB}$ transgenic mouse model of $\mathrm{OC}$ under obese and lean conditions. The significant finding of this study was that ONC201 inhibited ovarian tumor growth and reduced the ability of invasion via activation of ClpP induced oxidative stress pathways and inactivation of P13K/AKT/mTOR and MAPK pathways. Additionally, ONC201 induced apoptosis and cell cycle arrest in G1 phase in OC cells. Obesity induced by a HFD significantly promoted ovarian tumor growth as compared to LFD-fed mice. ONC201 significantly reduced tumor growth in both obese and lean KpB mice, accompanied by a decrease in $\mathrm{Ki}-67$ in tumor tissues and VEGF production in serum.

DRD2 Inhibition through genetic or pharmacological approaches has been shown to cause apoptosis and induce cell cycle arrest in leukemia, lung, colon, breast, endometrial, cervical, ovarian, pancreatic and brain cancer cells $(34,35,37-40)$. ONC201 has recently been shown to induce apoptosis via targeting DRD2 in a wide variety of different cancer types. The role of ONC201 mediated apoptotic pathways in its anti-tumorigenic activity have been shown to differ, depending on the cancer type. ONC201 was originally characterized as a selective antagonist of DRD2 to induce TRAIL and DR5 pathways, independent of p53 in cancer cells (41). In desmoplastic small round cell tumors, ONC201 increased the expression of TRAIL and DR5 and cell death via the extrinsic pathway of apoptosis (42). A recent study reported that the cytotoxicity of ONC201 was not dependent on either TRAIL death receptors or caspase cascades in breast cancer cells(43), whereas another study showed that ONC201 induced cell death that appeared to be through TRAIL-dependent and TRAIL-independent effects in breast cancer cells(44). Similar observations were noted in our recent study where we showed that ONC201 reduced BCL-2 expression and induced DR5 via extrinsic and intrinsic apoptotic pathways in USC cells (11). In agreement with these studies, we found that ONC201 treatment reduced BCL-XL and MCL-1 expression, induced DR5 upregulation and increased activity of cleaved caspase 3, 8 and 9 in OC cells. These findings indicate that ONC201-induced cell death can occur by both TRAIL-dependent and a TRAIL-independent mechanisms in 
OC cells, suggesting that the mechanism of ONC201 induced cell cytotoxicity may involve tissue- or cancer type-specific pathways in response to $\operatorname{ONC201}(11,16,41,45)$.

There is considerable evidence that DRD2 deficiency causes to endoplasmic reticulum (ER) stress which is required for the control of cancer cell growth $(34,46,47)$. The anti-cancer effect of ONC201 appears to be reliant on disrupting mitochondrial function, including inhibition of oxidative phosphorylation and reduction of the number of viable mitochondria (43). Several studies have reported that the tumor cell sensitivity to ONC201 is dependent on the induction of DR5 in an ATF4 and CHOP-dependent manner (48-50). In a phase I trial, ONC201 triggered the ISR along with induction of CHOP and TNFRSF10B in an ibrutinib-refractory mantle cell lymphoma patient following 16 days of treatment, which aligned with therapeutic exposure to ONC201 and target engagement in the tumor(14). Importantly, Graves et al recently applied an unbiased affinity proteomics approach and Ishizawa et al screened a small in-house library of 747 molecules approved for clinical use for malignant indications, and both discovered that ClpP is a critical molecular target that binds ONC201 in a direct and specific manner. Knockdown of ClpP by SiRNA decreased the response to ONC201 and blocked the expression of CHOP and the cytostatic effects induced by ONC201 in breast cancer cells. Activation of ClpP by ONC201 was associated directly with its anti-tumor activity through induction of ISR in vitro and in vivo $(7,8)$. Here, our results showed that ONC201 depolarized mitochondrial membranes and increased ROS levels in a dose-dependent manner, accompanied by an increase in the expression of ATF4, CHOP, PERK and Ero1-1a in OC cells, which are markers of oxidative stress related to apoptosis. We also confirmed that ONC201 treatment significantly induced the expression of ClpP in $\mathrm{OC}$ cells and KpB mice under obese and lean condition. SiRNA knockdown of ClpP in OC cells reduced the inhibitory effects of ONC201 although we did not observe a complete recovery of cell inhibition induced by ONC201 in ClpP knockdown cells. Overall, these studies suggest that: (1) cellular stress contributed to the anti-tumorigenic effects of ONC201 in addition to apoptosis and $\mathrm{G} 1$ cell cycle arrest in $\mathrm{OC}$ cells $(34,51)$, and (2) ClpP activation is partially responsible for the anti- tumorigenic activity of ONC201, and activation of ClpP provides a targeted approach to activate ER stress in cancer cells (52).

$\mathrm{PISK} / \mathrm{AKT} / \mathrm{mTOR}$ and MAPK/ERK pathways are responsible for mediating cell proliferation, invasion and tumorigenesis in OC. The PI3K/AKT/mTOR pathway is unregulated in approximately $70 \%$ of OC patients while activated MAPK pathway was more frequently expressed in low-grade $(81 \%)$ as compared with high-grade ovarian serous carcinomas (41\%)(53-55). The dysregulation of AKT and ERK signaling pathways in $\mathrm{OC}$ opens the possibility of actively targeting the signaling cascades, which might lead to superior anti-tumor activity. Several PI3K/AKT/mTOR inhibitors and MAPK/ERK targeted therapies are currently under evaluation in clinical trials against a variety of human cancers, including $\mathrm{OC}(56)$. In the current study, we found that ONC201 simultaneously reduced phosphorylation of AKT, S6 and p42/44 in OC cells in vitro. ONC201 strongly decreased the expression of phosphorylated S6 and p42/44 in the KpB OC mouse models under obese and lean conditions. The inhibition of PIK3CAVAKT/mTOR and MAPK pathways by ONC201 could be one of the major mechanisms by which it inhibited overall OC growth in the KpB mouse model. 
Peritoneal dissemination and local invasion by $\mathrm{OC}$ cells are involved in early steps of the metastatic process, which includes the transcriptional activation of ZEB1, TWIST, Slug and Snail, upregulation of Ecadherin and acquisition of a unique expression profile of EMT(57). The profile of EMT has been considered as a key hallmark for adhesion and invasion, and inhibition of EMT-related processes makes it particularly attractive for treatment of cancer(58). The regulatory role of DRD2 in adhesion and invasion has not been fully characterized, and the results remain controversial. Overexpression of DRD2 in patients with gastric cancer had shorter survival durations, and targeting DRD2 by thioridazine significantly inhibited cell proliferation in gastric cancer and reduced cell migration via suppression of EMT related genes in liver cancer $(32,59)$. However, increased expression of DRD2 in a neuroendocrine tumor patient was associated with longer survival, and activation of DRD2 by the DRD2 agonist BIM53097 reduced the ability of migration and invasion of human tumorous pituitary cells $(33,60)$. Similarly, fisetin as a DRD2 agonist suppressed liver cancer cell proliferation and reduced EMT through VEGFR1, p-ERK1/2, p38 and pJNK pathways (61). Recently, Wagner et al found that ONC201 is able to inhibit cancer cell invasion and exhibits a potent anti-metastatic effect in a TRAIL-dependent manner(62). Our previous work also confirmed that ONC201 inhibited adhesion and invasion, along with increasing the expression of Ecadherin and decreasing VEGF, N-cadherin and Snail expression in USC cells(11). In this current study, our results are consistent with our previous proposed mechanism of action of ONC201 involving EMT processes leading to inhibition of invasion in OC cells. We also demonstrated that ONC201 reduced the invasion index in organotypic 3D cultures as well as VEGF production in serum and ovarian tumors in the KpB mice. Moreover, pretreatment with NAC reversed ONC201-decreased VEGF and Snail levels, suggesting that ClpP or ClpP-induced oxidative stress may trigger the processes of adhesion and invasion induced by ONC201 in OC cells. These studies have provided insights into the mechanisms of the anti- metastatic effects of ONC201, which are dependent on oxidative stress.

\section{Conclusion}

Our study uncovered for the first time the potential anti-metastatic role of ONC201 in OC. ONC201 inhibited $\mathrm{OC}$ cell proliferation and tumor growth, which was associated with changes in expression of a constellation of proteins involved in apoptosis, cell cyclin, oxidative stress, angiogenesis, invasion, AMPK/mTOR and MAPK pathways. The mechanism of action of ONC201 to inhibit invasion is reliant on ClpP-mediated oxidative stress in OC. To date, ONC201 has entered multiple clinical trials in solid tumors and hematological malignancies. Our results have important preclinical implications indicating that ONC201 may be a promising agent in future OC clinical trials. Further studies are required to investigate the role of ClpP-mediated anti-proliferative and anti-metastatic effects in EMT and angiogenic pathways, and evaluate ClpP as a potential clinically useful biomarker in clinical trials of ONC201(52).

\section{Abbreviations}

DRD2: Dopamine receptor D2; DR5: Death receptor 5; OC: Ovarian cancer, ClpP: mitochondrial protease caseinolytic protease P; EMT: Epithelial-mesenchymal transition; $h T E R T$ : human telomerase reverse 
transcriptase; MAPK: Mitogen-activated protein kinase; VEGF: Vascular endothelial growth factor, TRAIL: tumor necrosis factor-related apoptosis-inducing ligand; FBS: fetal bovine serum; HFD: high fat diet; LFD: Low fat diet; ROS: Reactive oxygen species; USC: uterine serous carcinoma;

\section{Declarations}

\section{Availability of data and materials}

The datasets used and/or analyzed during the current study are available from the corresponding author on reasonable request

\section{Ethics approval and consent to participate}

All animals' experimental procedures were approved by Institutional Animal Care and Use Committee of University of North Carolina at Chapel Hill.

\section{Consent for publication}

Not applicable

\section{Competing interests}

VP and JEA are employees and stockholders of Oncoceutics. The other authors disclosed no potential conflicts of interest.

\section{Funding}

This work is supported by: (1) VLB: American Cancer Society (ACS) Research Scholar Grant - RSG CCE 128826. (2) VLB: NIH/NCI - R37CA226969. (3) JW: Beijing health system high-level health personnel training program fund (2014-3-073).

\section{Authors' contributions}

CZ and VLB conceived and designed the experiments. YF, JW, ZF, SRP, LW, AS, KT, YY and WK performed the main experiments and analyzed the data. YF, ZF and WS performed experiments in vivo. VP and JEA provided ONC201. CZ and VLB wrote the manuscript. All authors have read and approved the final manuscript. 


\section{Acknowledgements}

Not applicable

\section{References}

1. Siegel RL, Miller KD, Jemal A. Cancer statistics. 2020. CA: a cancer journal for clinicians. 2020;70(1):7-30. Epub 2020/01/09.

2. Iorio GC, Martini S, Arcadipane F, Ricardi U, Franco P. The role of radiotherapy in epithelial ovarian cancer: a literature overview. Medical oncology. 2019;36(7):64. Epub 2019/06/06.

3. Choi M, Fuller CD, Thomas CR Jr, Wang SJ. Conditional survival in ovarian cancer: results from the SEER dataset 1988-2001. Gynecol Oncol. 2008;109(2):203-9. Epub 2008/03/11.

4. Dai D, Zhou B, Xu W, Jin H, Wang X. CHFR Promoter Hypermethylation Is Associated with Gastric Cancer and Plays a Protective Role in Gastric Cancer Process. J Cancer. 2019;10(4):949-56. Epub 2019/03/12.

5. Allen JE, Kline CL, Prabhu WV, Wagner J, Ishizawa J, Madhukar N, et al. Discovery and clinical introduction of first-in-class imipridone ONC201. Oncotarget. 2016;7(45):74380-92. Epub 2016/09/08.

6. Prabhu VV, Talekar MK, Lulla AR, Kline CLB, Zhou L, Hall J, et al. Single agent and synergistic combinatorial efficacy of first-in-class small molecule imipridone ONC201 in hematological malignancies. Cell cycle. 2018;17(4):468-78. Epub 2017/11/22.

7. Graves PR, Aponte-Collazo LJ, Fennell EMJ, Graves AC, Hale AE, Dicheva N, et al. Mitochondrial Protease ClpP is a Target for the Anticancer Compounds ONC201 and Related Analogues. ACS chemical biology. 2019;14(5):1020-9. Epub 2019/04/26.

8. Ishizawa J, Zarabi SF, Davis RE, Halgas O, Nii T, Jitkova Y, et al. Mitochondrial ClpP-Mediated Proteolysis Induces Selective Cancer Cell Lethality. Cancer cell. 2019;35(5):721-37. e9. Epub 2019/05/06.

9. Wagner J, Kline CL, Ralff MD, Lev A, Lulla A, Zhou L, et al. Preclinical evaluation of the imipridone family, analogs of clinical stage anti-cancer small molecule ONC201, reveals potent anti-cancer effects of ONC212. Cell cycle. 2017;16(19):1790-9. Epub 2017/05/11.

10. Madhukar NS, Khade PK, Huang L, Gayvert K, Galletti G, Stogniew M, et al. A Bayesian machine learning approach for drug target identification using diverse data types. Nature communications. 2019;10(1):5221. Epub 2019/11/21.

11. Fang Z, Wang J, Clark LH, Sun W, Yin Y, Kong W, et al. ONC201 demonstrates anti-tumorigenic and anti-metastatic activity in uterine serous carcinoma in vitro. American journal of cancer research. 2018;8(8):1551-63. Epub 2018/09/14.

12. Arrillaga-Romany I, Chi AS, Allen JE, Oster W, Wen PY, Batchelor TT. A phase 2 study of the first imipridone ONC201, a selective DRD2 antagonist for oncology, administered every three weeks in 
recurrent glioblastoma. Oncotarget. 2017;8(45):79298-304. Epub 2017/11/08.

13. Karpel-Massler G, Siegelin MD. TIC10/ONC201-a potential therapeutic in glioblastoma. Translational cancer research. 2017;6(Suppl 9):1439-S40. Epub 2018/08/28.

14. Romaguera JE, Lee HJ, Tarapore R, Prabhu V, Allen J, Schalop L, et al. Integrated stress response and immune cell infiltration in an ibrutinib-refractory mantle cell lymphoma patient following ONC201 treatment. Br J Haematol. 2019;185(1):133-6. Epub 2018/05/10.

15. Chi AS, Tarapore RS, Hall MD, Shonka N, Gardner S, Umemura Y, et al. Pediatric and adult H3 K27Mmutant diffuse midline glioma treated with the selective DRD2 antagonist ONC201. Journal of neurooncology. 2019;145(1):97-105. Epub 2019/08/29.

16. Lev A, Lulla AR, Ross BC, Ralff MD, Makhov PB, Dicker DT, et al. ONC201 Targets AR and AR-V7 Signaling, Reduces PSA, and Synergizes with Everolimus in Prostate Cancer. Molecular cancer research: MCR. 2018;16(5):754-66. Epub 2018/03/29.

17. Choudhary GS, Al-Harbi S, Almasan A. Caspase-3 activation is a critical determinant of genotoxic stress-induced apoptosis. Methods in molecular biology. 2015;1219:1-9. Epub 2014/10/14.

18. Wysham WZ, Roque DR, Han J, Zhang L, Guo H, Gehrig PA, et al. Effects of Fatty Acid Synthase Inhibition by Orlistat on Proliferation of Endometrial Cancer Cell Lines. Target Oncol. 2016;11(6):763-9. Epub 2016/05/18.

19. Jafari SM, Joshaghani HR, Panjehpour M, Aghaei M, Zargar Balajam N. Apoptosis and cell cycle regulatory effects of adenosine by modulation of GLI-1 and ERK1/2 pathways in CD44(+) and CD24(-) breast cancer stem cells. Cell proliferation. 2017;50(4). Epub 2017/04/04.

20. Perry SW, Norman JP, Barbieri J, Brown EB, Gelbard HA. Mitochondrial membrane potential probes and the proton gradient: a practical usage guide. Biotechniques. 2011;50(2):98-115. Epub 2011/04/14.

21. Jenei V, Nystrom ML, Thomas GJ. Measuring invasion in an organotypic model. Methods in molecular biology. 2011;769:223-32. Epub 2011/07/13.

22. Suri A, Sheng $X$, Schuler KM, Zhong $Y$, Han $X$, Jones HM, et al. The effect of celecoxib on tumor growth in ovarian cancer cells and a genetically engineered mouse model of serous ovarian cancer. Oncotarget. 2016;7(26):39582-94. Epub 2016/10/27.

23. Han J, Wysham WZ, Zhong Y, Guo H, Zhang L, Malloy KM, et al. Increased efficacy of metformin corresponds to differential metabolic effects in the ovarian tumors from obese versus lean mice. Oncotarget. 2017;8(67):110965-82. Epub 2018/01/18.

24. Szabova L, Yin C, Bupp S, Guerin TM, Schlomer JJ, Householder DB, et al. Perturbation of Rb, p53, and Brca1 or Brca2 cooperate in inducing metastatic serous epithelial ovarian cancer. Cancer Res. 2012;72(16):4141-53. Epub 2012/05/24.

25. Crowley LC, Christensen ME, Waterhouse NJ. Measuring Survival of Adherent Cells with the ColonyForming Assay. Cold Spring Harbor protocols. 2016;2016(8). Epub 2016/08/03.

26. Makowski L, Zhou C, Zhong Y, Kuan PF, Fan C, Sampey BP, et al. Obesity increases tumor aggressiveness in a genetically engineered mouse model of serous ovarian cancer. Gynecol Oncol. 
2014;133(1):90-7. Epub 2014/04/01.

27. Voltan R, Secchiero P, Casciano F, Milani D, Zauli G, Tisato V. Redox signaling and oxidative stress: Cross talk with TNF-related apoptosis inducing ligand activity. The international journal of biochemistry \& cell biology. 2016;81(Pt B):364 - 74. Epub 2016/10/01.

28. Hill BS, Sarnella A, D'Avino G, Zannetti A. Recruitment of stromal cells into tumour microenvironment promote the metastatic spread of breast cancer. Seminars in cancer biology. 2019. Epub 2019/08/05.

29. Nystrom ML, Thomas GJ, Stone M, Mackenzie IC, Hart IR, Marshall JF. Development of a quantitative method to analyse tumour cell invasion in organotypic culture. J Pathol. 2005;205(4):468-75. Epub 2005/02/03.

30. Wang X, Wang ZB, Luo C, Mao XY, Li X, Yin JY, et al. The Prospective Value of Dopamine Receptors on Bio-Behavior of Tumor. J Cancer. 2019;10(7):1622-32. Epub 2019/06/18.

31. Roney MSI, Park SK. Antipsychotic dopamine receptor antagonists, cancer, and cancer stem cells. Arch Pharm Res. 2018;41(4):384-408. Epub 2018/03/21.

32. Mu J, Huang W, Tan Z, Li M, Zhang L, Ding Q, et al. Dopamine receptor D2 is correlated with gastric cancer prognosis. Oncology letters. 2017;13(3):1223-7. Epub 2017/04/30.

33. Grossrubatscher E, Veronese S, Ciaramella PD, Pugliese R, Boniardi M, De Carlis L, et al. High expression of dopamine receptor subtype 2 in a large series of neuroendocrine tumors. Cancer Biol Ther. 2008;7(12):1970-8. Epub 2008/11/05.

34. Jandaghi P, Najafabadi HS, Bauer AS, Papadakis Al, Fassan M, Hall A, et al. Expression of DRD2 Is Increased in Human Pancreatic Ductal Adenocarcinoma and Inhibitors Slow Tumor Growth in Mice. Gastroenterology. 2016;151(6):1218-31. Epub 2016/10/30.

35. Yong M, Yu T, Tian S, Liu S, Xu J, Hu J, et al. DR2 blocker thioridazine: A promising drug for ovarian cancer therapy. Oncology letters. 2017;14(6):8171-7. Epub 2018/01/19.

36. Prabhu WV, Madhukar NS, Gilvary C, Kline CLB, Oster S, El-Deiry WS, et al. Dopamine Receptor D5 is a Modulator of Tumor Response to Dopamine Receptor D2 Antagonism. Clinical cancer research: an official journal of the American Association for Cancer Research. 2019;25(7):2305-13. Epub 2018/12/19.

37. Tegowski M, Fan C, Baldwin AS. Thioridazine inhibits self-renewal in breast cancer cells via DRD2dependent STAT3 inhibition, but induces a G1 arrest independent of DRD2. J Biol Chem. 2018;293(41):15977-90. Epub 2018/08/23.

38. Meng Q, Sun $X$, Wang J, Wang $Y$, Wang L. The important application of thioridazine in the endometrial cancer. American journal of translational research. 2016;8(6):2767-75. Epub 2016/07/12.

39. Mao M, Yu T, Hu J, Hu L. Dopamine D2 receptor blocker thioridazine induces cell death in human uterine cervical carcinoma cell line SiHa. J Obstet Gynaecol Res. 2015;41(8):1240-5. Epub 2015/04/03. 
40. Sheikhpour M, Ahangari G, Sadeghizadeh M, Deezagi A. A novel report of apoptosis in human lung carcinoma cells using selective agonist of D2-like dopamine receptors: a new approach for the treatment of human non-small cell lung cancer. Int J ImmunoPathol Pharmacol. 2013;26(2):393402. Epub 2013/06/13.

41. Talekar MK, Allen JE, Dicker DT, El-Deiry WS. ONC201 induces cell death in pediatric non-Hodgkin's lymphoma cells. Cell Cycle. 2015;14(15):2422-8. Epub 2015/06/02.

42. Hayes-Jordan AA, Ma X, Menegaz BA, Lamhamedi-Cherradi SE, Kingsley CV, Benson JA, et al. Efficacy of ONC201 in Desmoplastic Small Round Cell Tumor. Neoplasia. 2018;20(5):524-32. Epub 2018/04/08.

43. Greer YE, Porat-Shliom N, Nagashima K, Stuelten C, Crooks D, Koparde VN, et al. ONC201 kills breast cancer cells in vitro by targeting mitochondria. Oncotarget. 2018;9(26):18454-79. Epub 2018/05/03.

44. Ralff MD, Kline CLB, Kucukkase OC, Wagner J, Lim B, Dicker DT, et al. ONC201 Demonstrates Antitumor Effects in Both Triple-Negative and Non-Triple-Negative Breast Cancers through TRAILDependent and TRAIL-Independent Mechanisms. Mol Cancer Ther. 2017;16(7):1290-8. Epub 2017/04/21.

45. Endo Greer Y, Lipkowitz S. ONC201: Stressing tumors to death. Sci Signal. 2016;9(415):fs1. Epub 2016/02/18.

46. Tinsley RB, Bye CR, Parish CL, Tziotis-Vais A, George S, Culvenor JG, et al. Dopamine D2 receptor knockout mice develop features of Parkinson disease. Ann Neurol. 2009;66(4):472-84. Epub 2009/10/23.

47. Yang Y, Zhang Y, Cuevas S, Villar VA, Escano C. L DA, et al. Paraoxonase 2 decreases renal reactive oxygen species production, lowers blood pressure, and mediates dopamine D2 receptor-induced inhibition of NADPH oxidase. Free Radic Biol Med. 2012;53(3):437-46. Epub 2012/05/29.

48. Allen JE, Krigsfeld G, Mayes PA, Patel L, Dicker DT, Patel AS, et al. Dual inactivation of Akt and ERK by TIC10 signals Foxo3a nuclear translocation, TRAIL gene induction, and potent antitumor effects. Science translational medicine. 2013;5(171):171 ra17. Epub 2013/02/08.

49. Kline CL, Van den Heuvel AP, Allen JE, Prabhu VV, Dicker DT, El-Deiry WS. ONC201 kills solid tumor cells by triggering an integrated stress response dependent on ATF4 activation by specific elF2alpha kinases. Sci Signal. 2016;9(415):ra18. Epub 2016/02/18.

50. Ishizawa J, Kojima K, Chachad D, Ruvolo P, Ruvolo V, Jacamo RO, et al. ATF4 induction through an atypical integrated stress response to ONC201 triggers p53-independent apoptosis in hematological malignancies. Sci Signal. 2016;9(415):ra17. Epub 2016/02/18.

51. Weissenrieder JS, Neighbors JD, Mailman RB, Hohl RJ. Cancer and the Dopamine D2 Receptor: A Pharmacological Perspective. J Pharmacol Exp Ther. 2019;370(1):111-26. Epub 2019/04/20.

52. Wang S, Dougan DA. The Direct Molecular Target for Imipridone ONC201 Is Finally Established. Cancer cell. 2019;35(5):707-8. Epub 2019/05/16.

53. Hsu CY, Bristow R, Cha MS, Wang BG, Ho CL, Kurman RJ, et al. Characterization of active mitogenactivated protein kinase in ovarian serous carcinomas. Clinical cancer research: an official journal of 
the American Association for Cancer Research. 2004;10(19):6432-6. Epub 2004/10/12.

54. Cheaib B, Auguste A, Leary A. The PI3K/Akt/mTOR pathway in ovarian cancer: therapeutic opportunities and challenges. Chinese journal of cancer. 2015;34(1):4-16. Epub 2015/01/06.

55. Gasparri ML, Bardhi E, Ruscito I, Papadia A, Farooqi AA, Marchetti C, et al. PI3K/AKT/mTOR Pathway in Ovarian Cancer Treatment: Are We on the Right Track? Geburtshilfe Frauenheilkd. 2017;77(10):1095-103. Epub 2017/11/03.

56. Brasseur K, Gevry N, Asselin E. Chemoresistance and targeted therapies in ovarian and endometrial cancers. Oncotarget. 2017;8(3):4008-42. Epub 2016/12/23.

57. Moffitt L, Karimnia N, Stephens A, Bilandzic M. Therapeutic Targeting of Collective Invasion in Ovarian Cancer. International journal of molecular sciences. 2019;20(6). Epub 2019/03/27.

58. Mittal V. Epithelial Mesenchymal Transition in Tumor Metastasis. Annual review of pathology. 2018;13:395-412. Epub 2018/02/08.

59. Lu M, Li J, Luo Z, Zhang S, Xue S, Wang K, et al. Roles of dopamine receptors and their antagonist thioridazine in hepatoma metastasis. OncoTargets therapy. 2015;8:1543-52. Epub 2015/07/01.

60. Peverelli E, Giardino E, Treppiedi D, Locatelli M, Vaira V, Ferrero S, et al. Dopamine receptor type 2 (DRD2) inhibits migration and invasion of human tumorous pituitary cells through ROCK-mediated cofilin inactivation. Cancer letters. 2016;381(2):279-86. Epub 2016/08/16.

61. Liu XF, Long HJ, Miao XY, Liu GL, Yao HL. Fisetin inhibits liver cancer growth in a mouse model: Relation to dopamine receptor. Oncol Rep. 2017;38(1):53-62. Epub 2017/06/01.

62. Wagner J, Kline CL, Zhou L, Campbell KS, MacFarlane AW, Olszanski AJ, et al. Dose intensification of TRAIL-inducing ONC201 inhibits metastasis and promotes intratumoral NK cell recruitment. J Clin Investig. 2018;128(6):2325-38. Epub 2018/03/14.

\section{Figures}



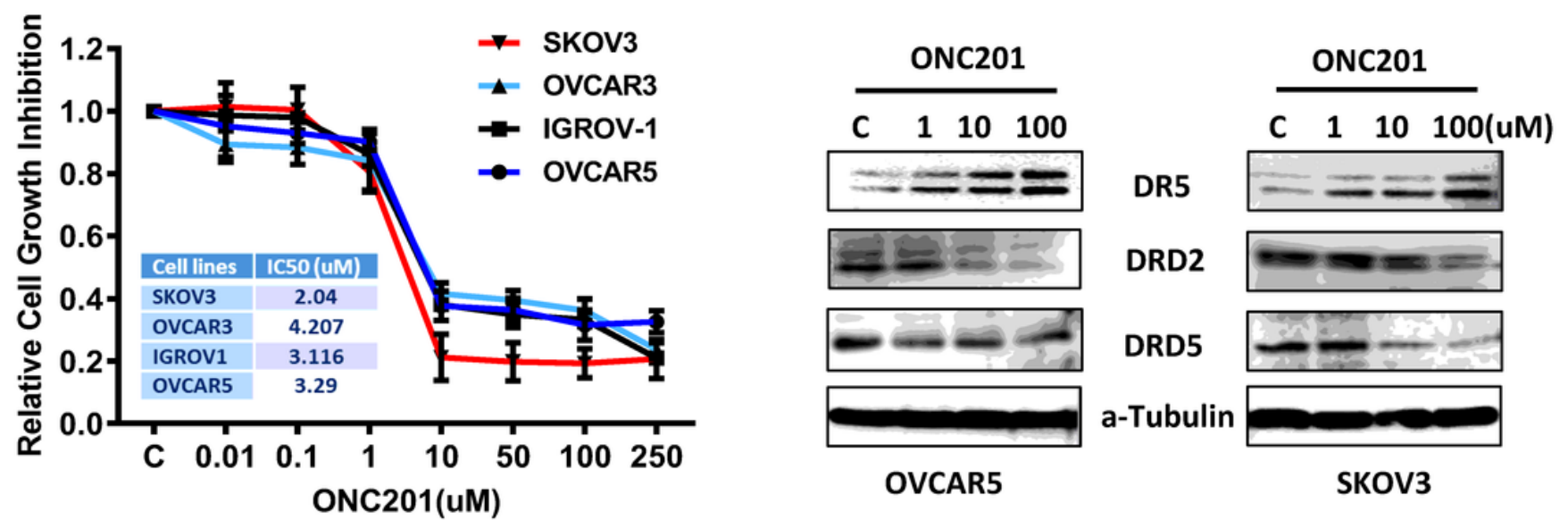

B
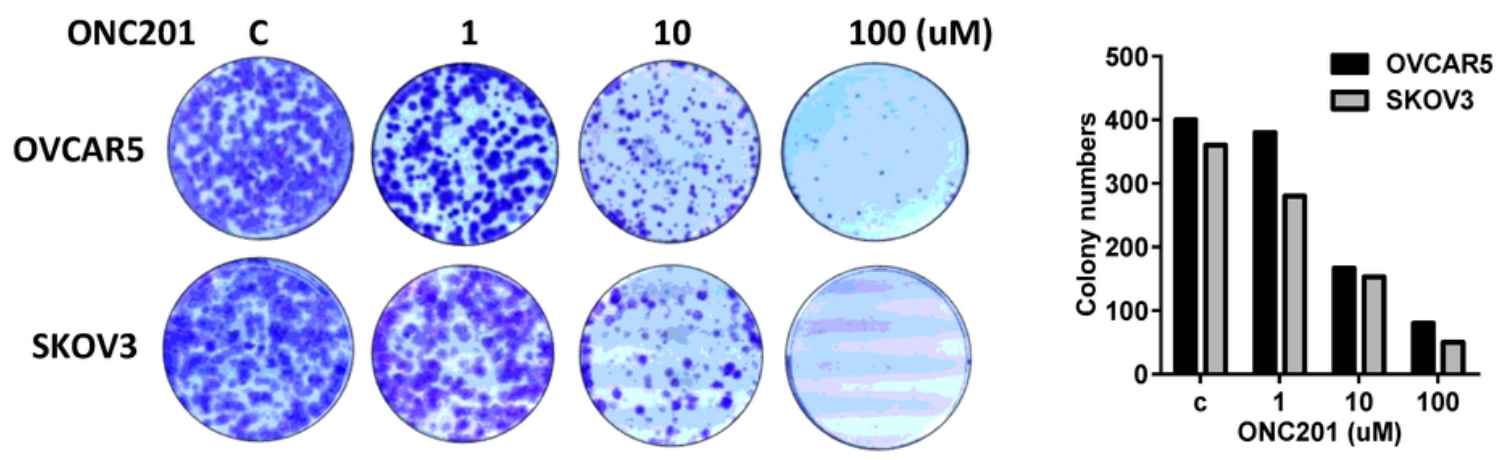

Figure 1

ONC201 inhibited cell viability and colony formation. The OVCAR5, OVCAR3, IGOV-1 and SKOV3 in 96 well plates were treated with increasing concentrations of ONC201 for 72 hours and subjected to the MTT assay. ONC210 significantly inhibited cell growth in a dose- dependent manner in all cell lines. Similar results were obtained from three independent experiments (A). ONC201 inhibited colony forming ability of OVCAR5 and SKOV3 cells. Cells were incubated with ONC201 for 48 hours, then cell were culture in drugfree media for 12 days $(B)$. The images and bar chart represented results of one experiment. Western Blotting was used to evaluate the effect of ONC210 on expression of DRD2, DRD5 and DR5 in the OVCAR5 and SKOV3 cells. ONC201 decreased the expression of DRD2 and DRD5 and increased the expression of DR5 in a dose-dependent manner in both cell lines (C). 
C
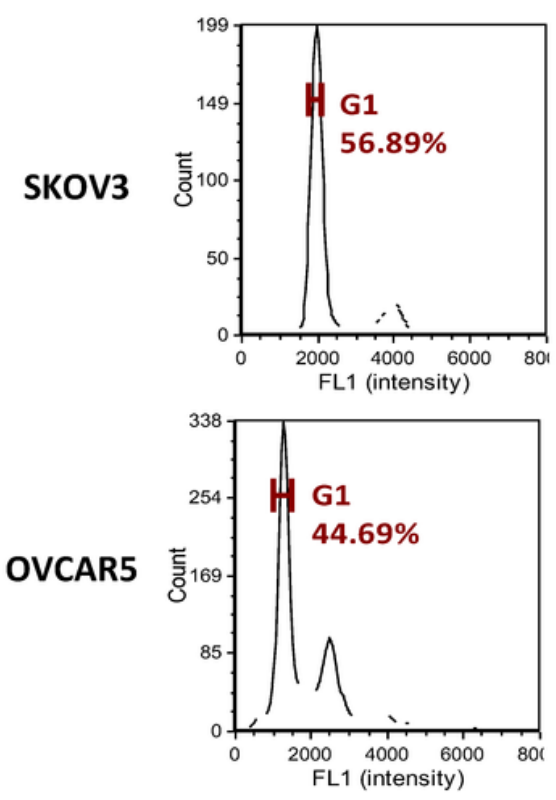

1
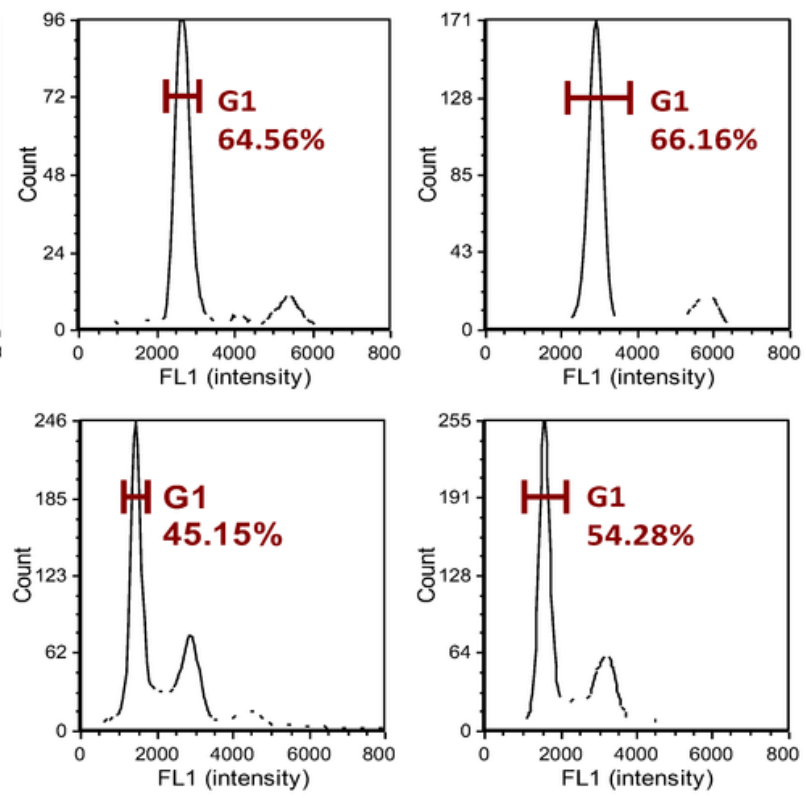

10

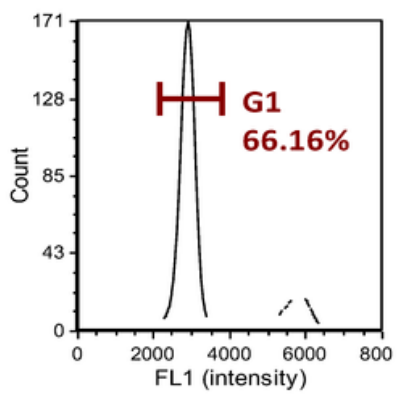

100 (uM)
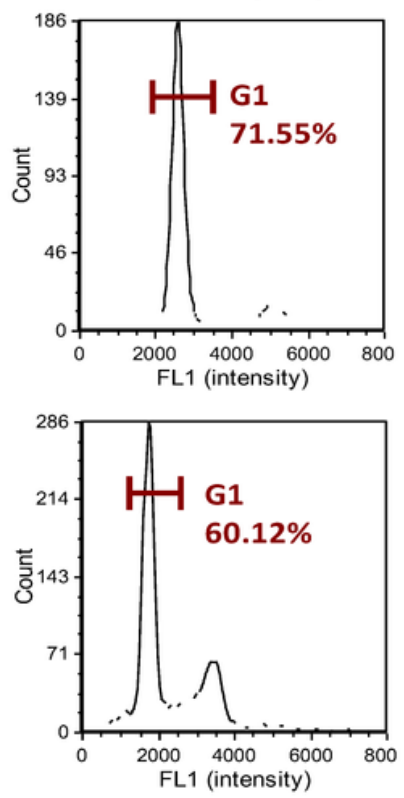

B
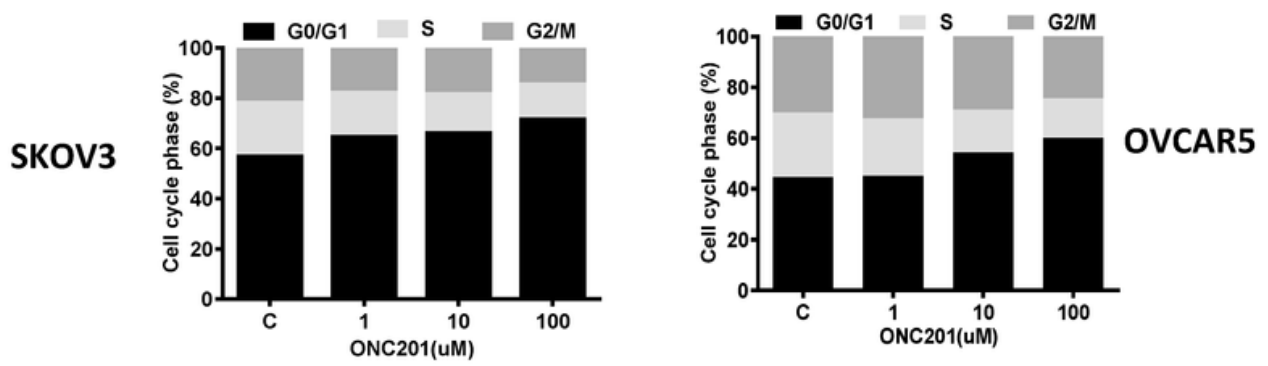

C

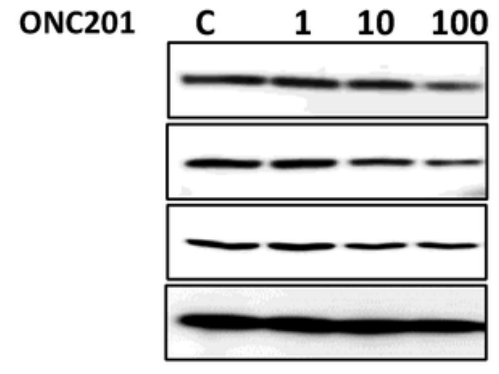

SKOV3

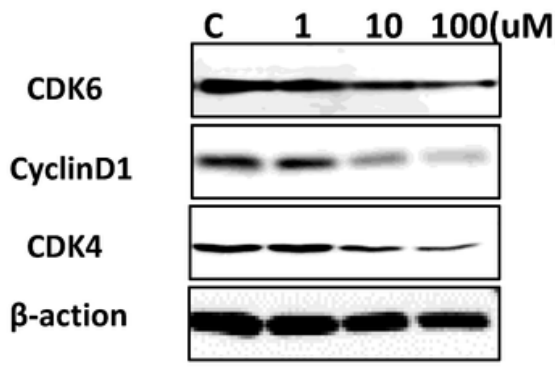

OVCAR5

Figure 2

Induction of cell cycle G1 arrest by ONC201 is dose-dependent. The OVCAR5 and SKOV3 cells were incubated with indicated doses of ONC201 for 36 hours. Cell cycle progression was assessed by Cellometer. Cell cycle $\mathrm{G} 1$ arrest was found in both cell lines in a dose-dependent manner (A and B). The cells were exposed to 0-100 um ONC201 for 36 hours prior to western blotting for the detection CDK4, CDK6 and Cyclin D1. ONC201 decreased the expression of CDK4, CDK6 and Cyclin D1 in both cell lines. $\beta$ actin was used as loading control (C). The results shown are one of three independent experiments. 
A

ONC201

OVCAR5

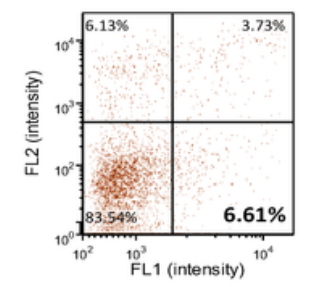

PI

SKOV3

B

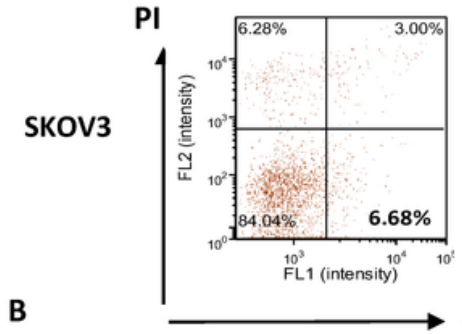

1
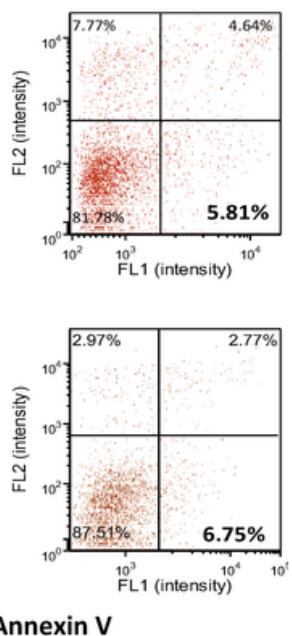

Annexin V
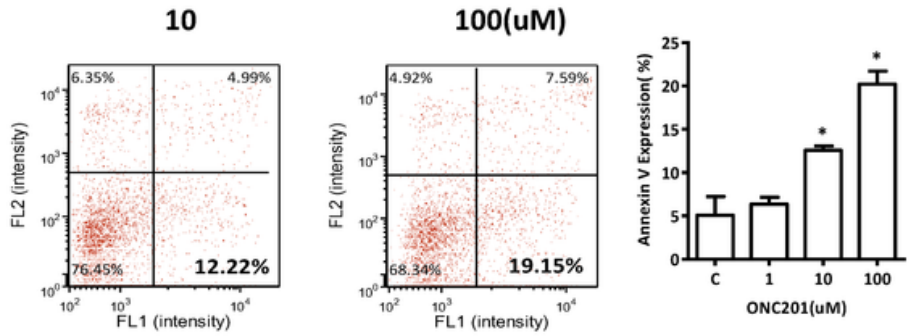
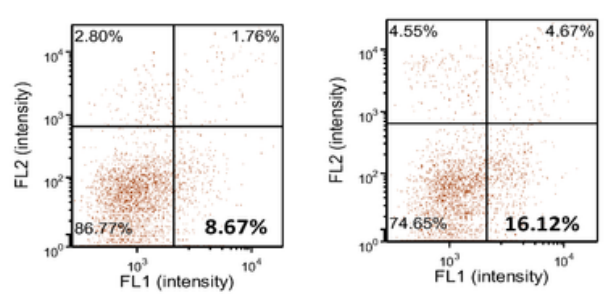

C
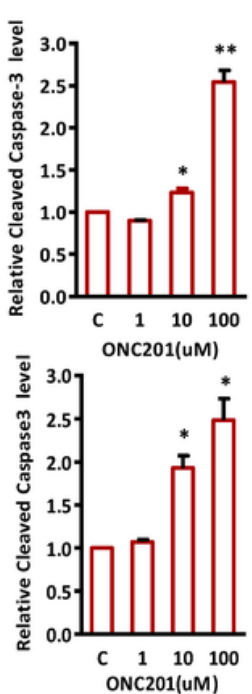
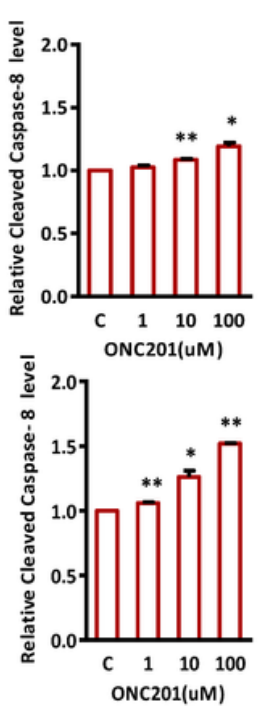

C 110100 (uM)

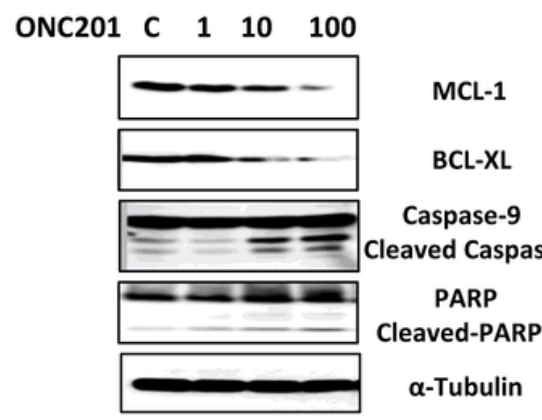

OVCAR5
SKOV3

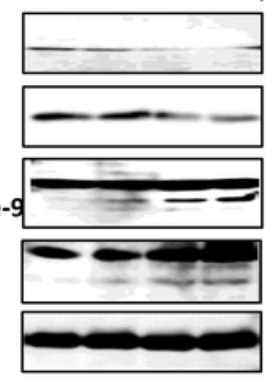

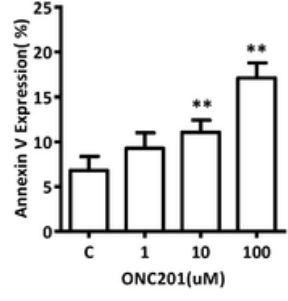
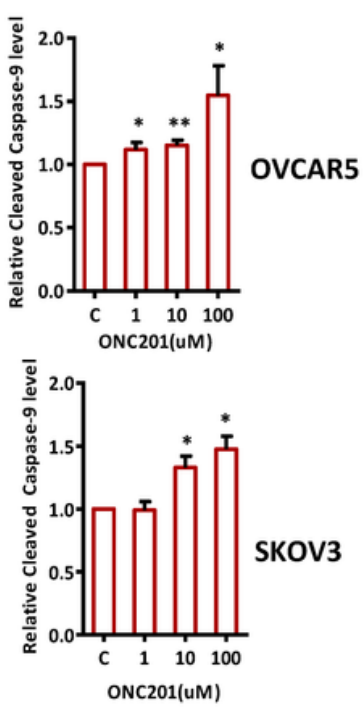

Figure 3

ONC201 induced apoptosis in OC cells. OVCAR5 and SKOV3 cells were incubated for 30 hours with the indicated amount of ONC201. Annexin V was analyzed by Cellometer (A). Total cell lysates were prepared and analyzed by western blotting for MCL-1, BCL-XL, Cleaved caspase 9 and PARP (B). Cleaved caspase 3,8 and 9 activity was assayed by ELISA assay. ONC201 induced the activity of the cleaved caspases 3 , 8 and 9 in both cell lines after treatment with ONC201 for 8-12 hours (C). The results are shown as the mean \pm SD and are representative of three independent experiments. ${ }^{*} p<0.05,{ }^{*} p<0.01$. 
A

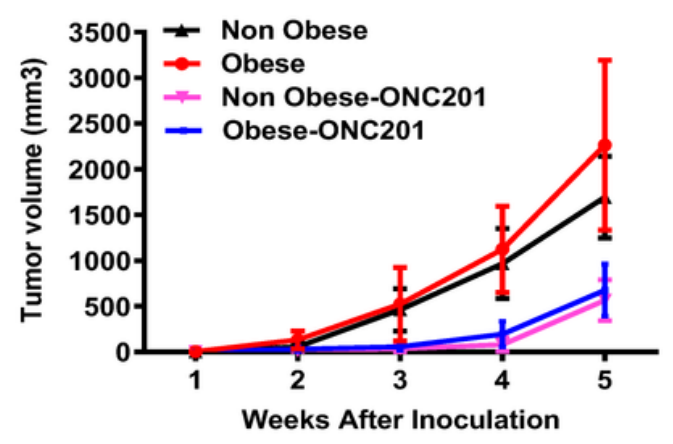

B

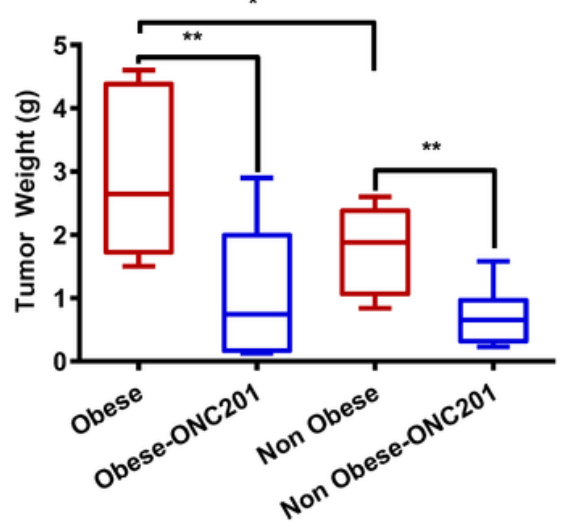

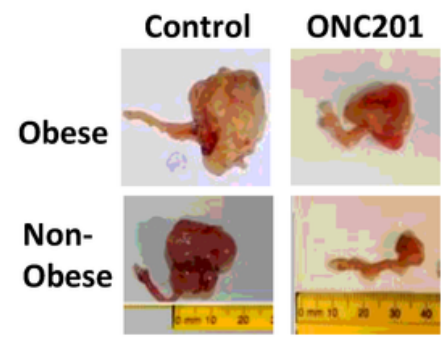

C

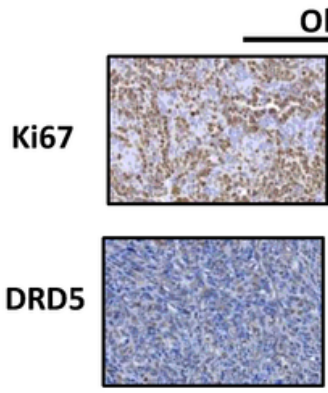

Control
Obese

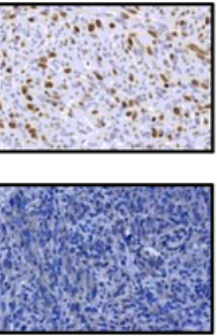

ONC201
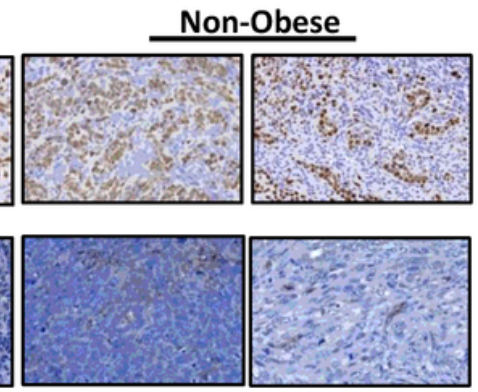

Control

ONC201
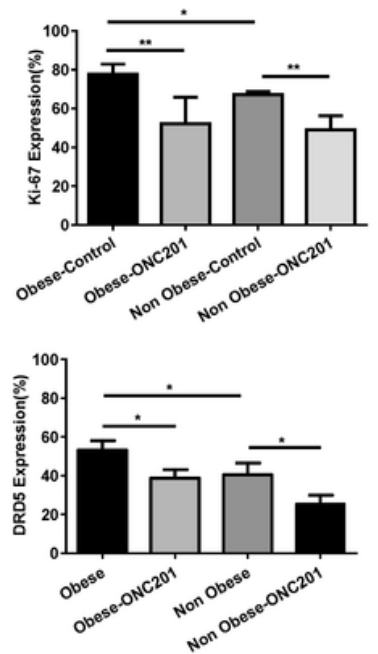

Figure 4

ONC201 inhibited tumor growth in KpB mouse model of OC. Obese or lean KpB mice were treated with ONC201 (130 mg/kg, weekly) or vehicle for 4 weeks. Tumor volumes of ovarian tumors were measured weekly. The mean tumor volume was reduced in obese or lean mice treated with ONC201 (A). Tumor weight measurements of $\mathrm{KpB}$ mice were recorded at the time of sacrifice (B). The expression of DRD5 and Ki67 was assessed by immunohistochemistry analysis in ovarian tumors following 0NC201 treatment. The results showed that ONC201 decreased the expression of Ki-67 and DRD5 in the ovarian tumor tissues under obese and lean conditions (C). * $p<0.05 ;{ }^{* *} p<0.01$ 
A
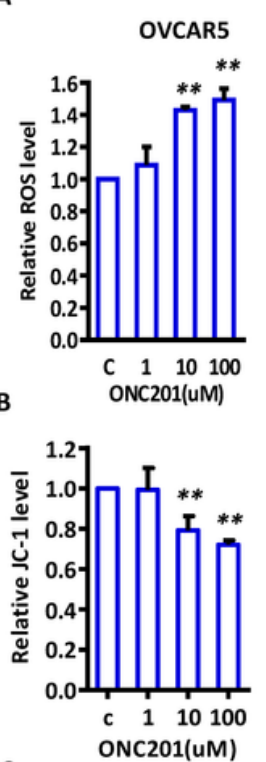

C

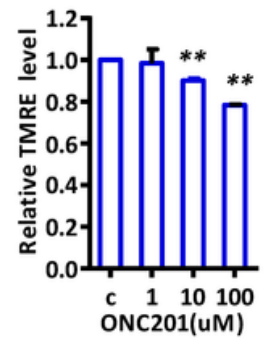

SKOV3
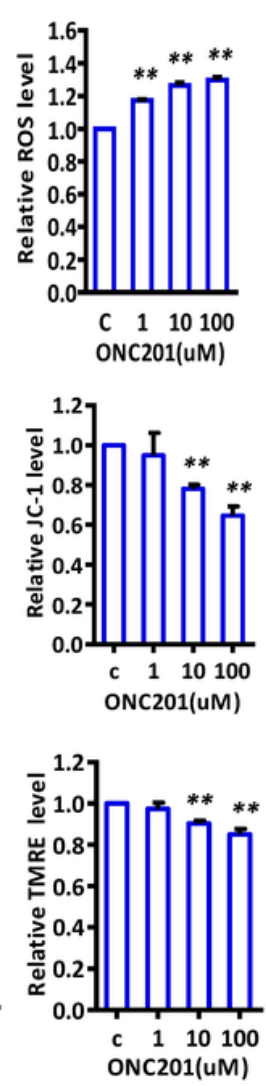

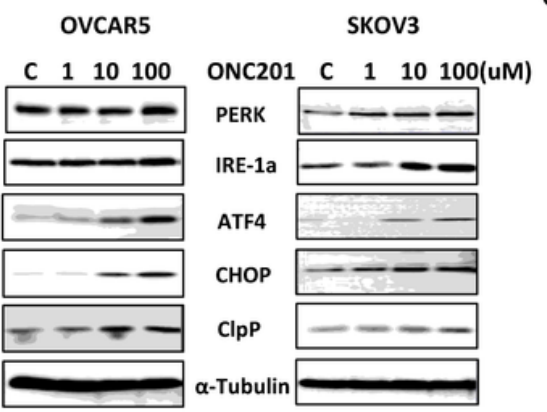

$\mathbf{E}$

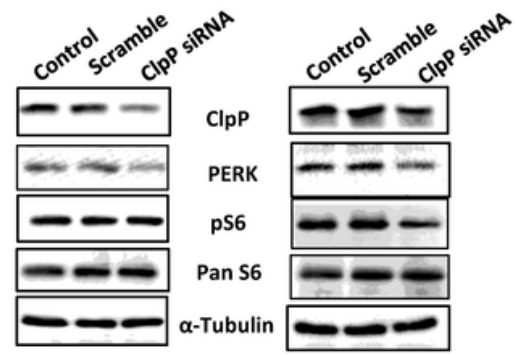

$\mathbf{F}$

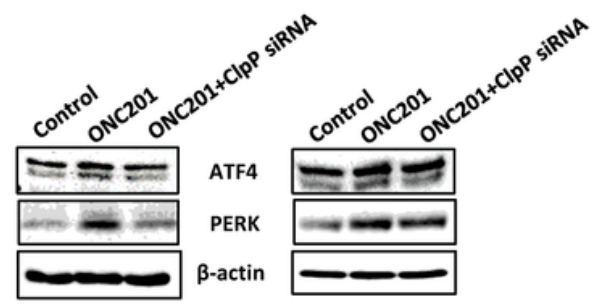

OVCAR5

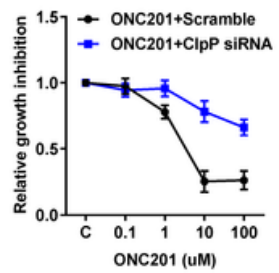

H
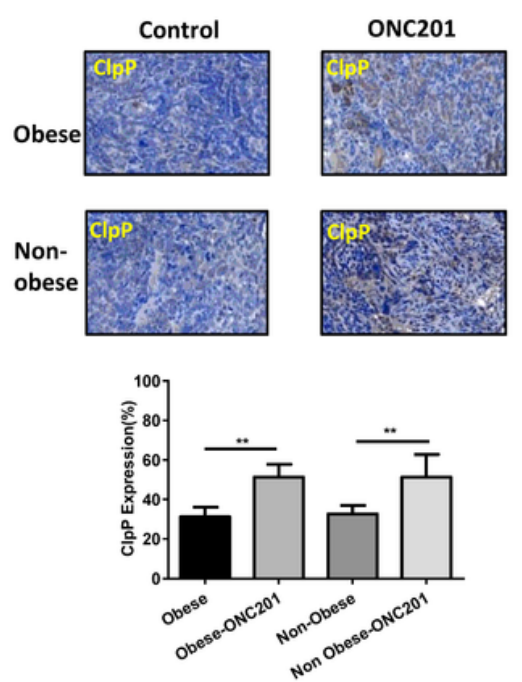

Figure 5

ONC201 induced ER stress in OC cells. Cell stress analysis was done on OVCAR5 and SKOV3 cells treated with ONC201. ROS, JC-1 and TMRE products were measured by ELISA assays. ONC201 significantly increased the levels of ROS and decreased mitochondrial membrane potential in both cell lines compared to the control-treated cells (A-C). ONC201 increased the expression of cellular stress related proteins including PERK, IRE-1a, ATF4, CHOP and ClpP in both cell lines after 24 hours of treatment (D). Knockdown ClpP reduced the expression of PERK and phosphorylated S6 in both cells (E). ClpP siRNA decreased ONC201 mediated ATF4 and PERK expression and ONC201 induced cell inhibition in both cells ( $F$ and G). In KpB mice, ONC201 also induced ClpP expression in the ovarian tumors under obese and lean conditions, as measured by $\mathrm{IHC}(\mathrm{H})$. 
A

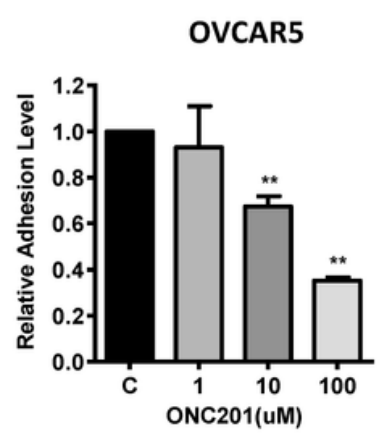

C
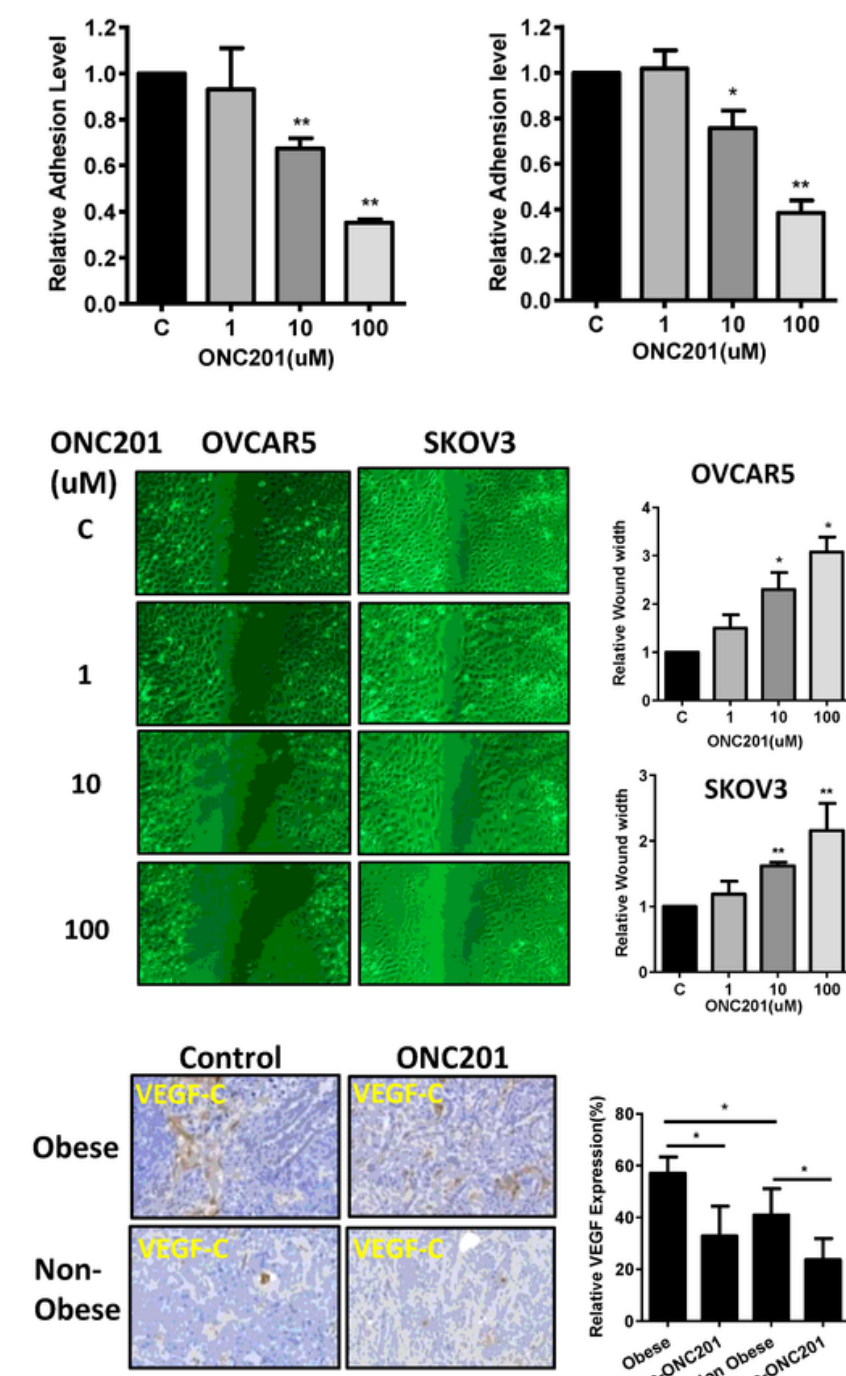

SKOV3
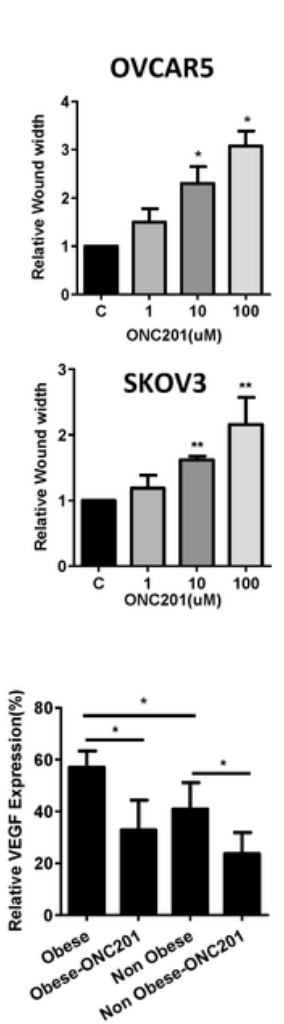

B

OVCAR5

SKOV3
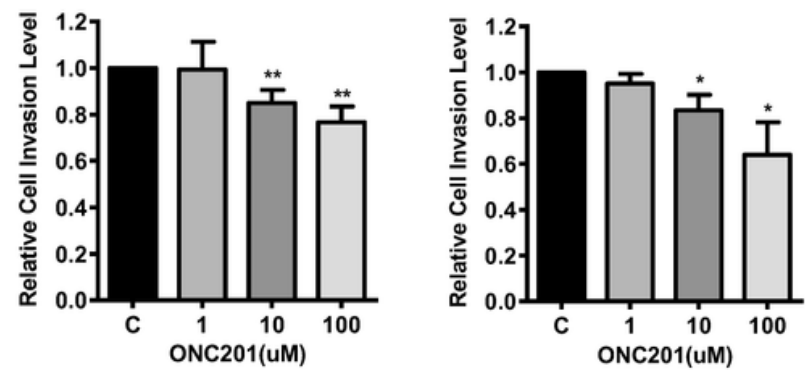

D

Control

ONC201

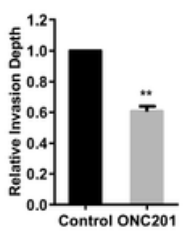

E

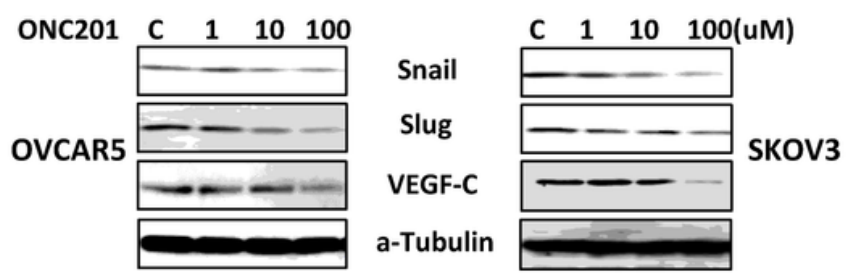

G

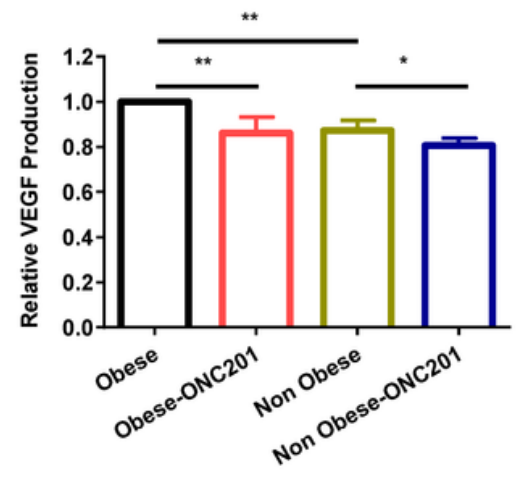

Figure 6

ONC201 inhibited adhesion and invasion. The OVCAR5 and SKOV3 cells were treated with ONC201 at a range of doses from 1-100 $\mu \mathrm{M}$. laminin-1 assay was used to assess adhesion after 2 hour of treatment with ONC201 (A). Invasion was examined by transwell assay after 4 hours of treatment with ONC201 (B). Migration was assessed by wound healing assay after treatment with ONC201 for 48 hours (C). Organotypic 3D co-cultures results showed that ONC201 significantly reduced the ability of adhesion, migration and invasion in OVCAR5 cells (D). Western blotting found that ONC201 reduced the expression of VEGF, Snail and Slug in both cell lines (E). The obese and lean KpB mice were treated with ONC201 for 4 weeks. IHC results showed that ONC201 reduced VEGF expression in both obese and lean mice (F). Obesity was associated with increased serum VEGF production as compared to lean mice, and ONC201 reduced the production of VEGF in serum under obese and lean conditions (G). 
A

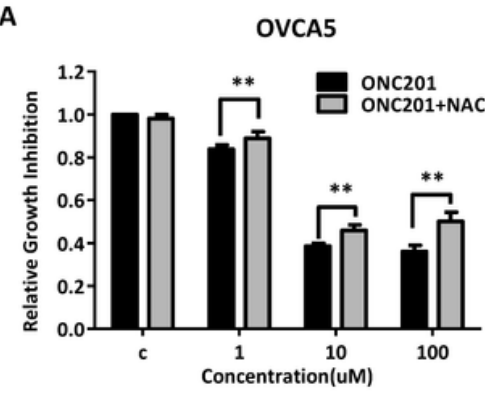

C

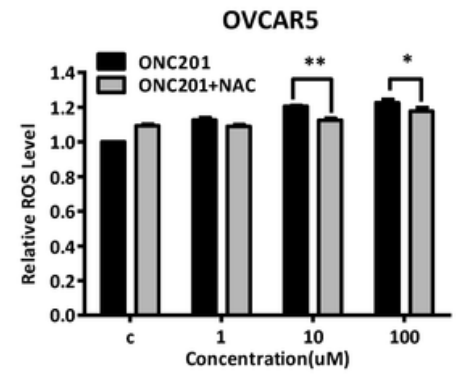

E

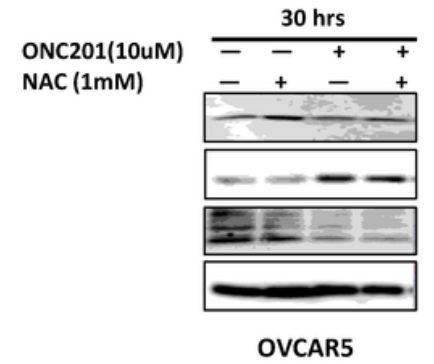

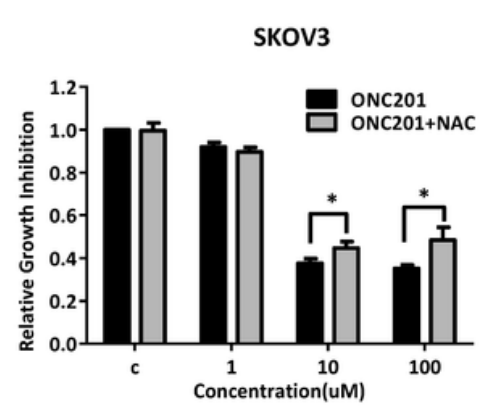

SKOV3
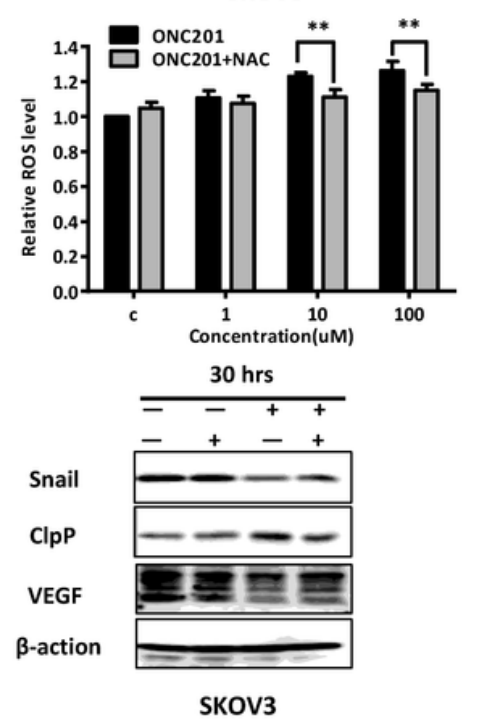

B

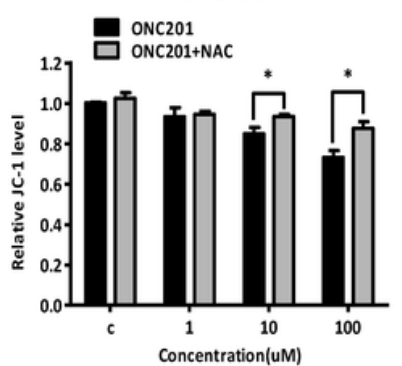

D

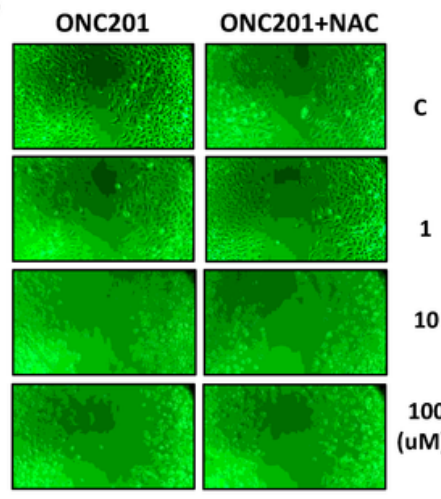

OVCAR5

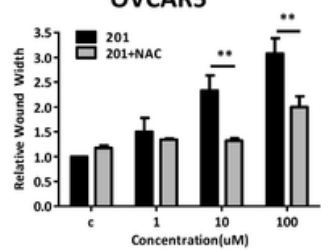

SKOV3
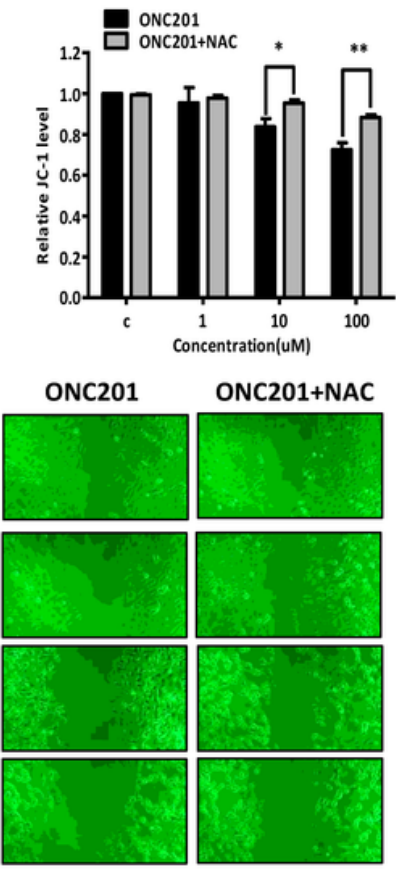

SKOV3

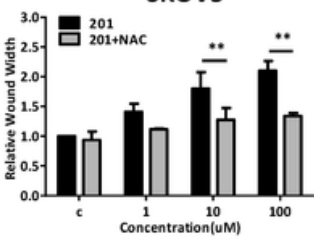

Figure 7

ONC201 inhibited adhesion and invasion through cellular stress pathways in OC cells. Pre-treatment of the OCCAR5 and SKOV3 cells with $1 \mathrm{mM}$ NAC partially blocked inhibition of ONC201-mediated proliferation in both cell line (A). Similarly, pre-treatment with NCA for 6 hours in OCVAR5 and SKOV3 cells significantly reduced ROS levels and recovered mitochondrial membrane potential induced by ONC201 (B and C). The cells were pre-treated with NAC for 6 hours, followed by treatment with ONC201 for $48 \mathrm{hr}$. Wound healing was observed by a microscope with phase contrast. ONC201 decreased cell migration, and NAC effectively reversed ONC201-inhibited cell migration in the OVCAR5 and SKOV3 cells. All images were obtained from three independent experiments (D). Western blotting analysis for ClpP, VEGF and Snail on protein lysates from OVCAR5 and SKOV3 cells treated with NAC or ONC201 for 24 hours. NAC partially inhibited ONC201-induced decreases in Snail and VEGF and increases in ClpP in both cells (E). *P $<0.05, * * \mathrm{P}<0.01$. 
A OVCAR5

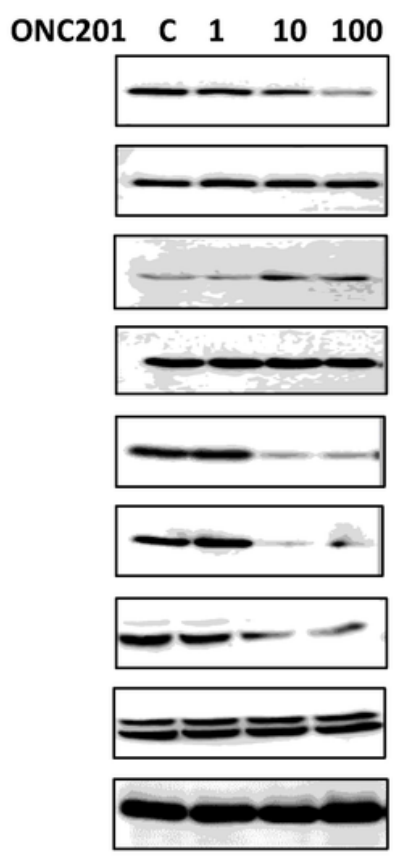

SKOV3

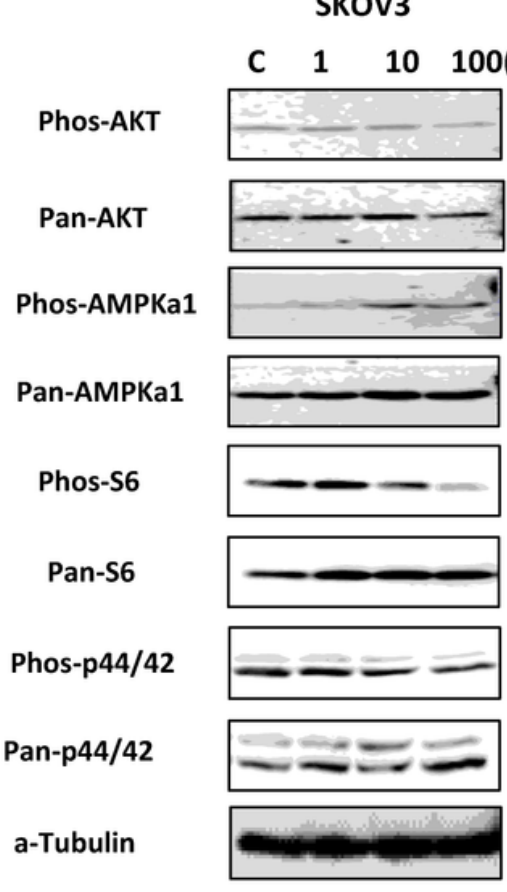

B

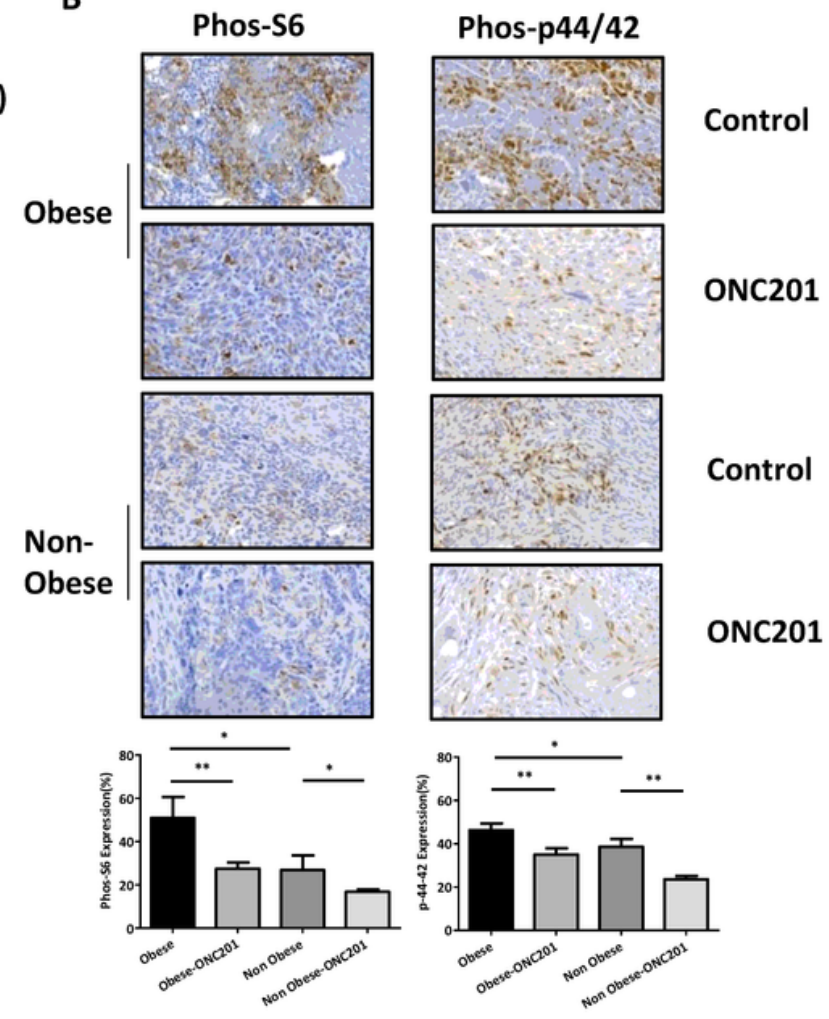

Figure 8

ONC201 activated AMPK and inactivated AKT/mTOR and MAPK pathways. The OVCAR5 and SKOV3 ovarian cell lines were incubated with ONC201 at various concentrations for 24 hours. Western blotting results indicated ONC201 decreased the expression of phosphorylated Akt, S6, p42/44, and increased the phosphorylated AMPK expression in both cell lines (A). The expression of phosphorylated AMPK1, Akt, S6 and p42/44 was analyzed by western blotting. The KpB mice were treated with ONC201 for 4 weeks. IHC results showed that obesity increased expression of phosphorylation of S6 and p42/44, and ONC201 significantly decreased the expression of phosphorylated p-S6 and p42/44 in ovarian tumors under obese and lean conditions (B) 